Article

\title{
Synthesis and Antimicrobial Evaluation of Some New Organic Tellurium Compounds Based on Pyrazole Derivatives
}

\author{
Asmaa B. Sabti ${ }^{1}$, Adil A. Al-Fregi ${ }^{2, *}$ and Majeed Y. Yousif ${ }^{2}$ \\ 1 Department of Chemistry, College of Science, University of Misan, Basrah 62001, Iraq; \\ asmaabadr86.ab@gmail.com \\ 2 Department of Chemistry, College of Science, University of Basrah, Basrah 61004, Iraq; \\ majeedyousef@gmail.com \\ * Correspondence: dr.adilalfregi@gmail.com or dr.adilalfregi@uobasrah.edu.iq
}

Academic Editor: Julien Furrer

Received: 23 May 2020; Accepted: 22 June 2020; Published: 29 July 2020

Abstract: A novel series of organic tellurium compounds based on pyrazole derivatives with a general formula of $\mathrm{ArTeBr}_{3}$ and $\mathrm{Ar}_{2} \mathrm{TeBr}_{2}$ [Ar $=$ 2-(3-(4-substituted phenyl)-5-(2-chlorophenyl) -1H-pyrazol-1-yl)-3,5-dinitrophenyl] were obtained by the refluxing of corresponding aryl mercuric chlorides with $\mathrm{TeBr}_{4}$ in two different mole ratio of 1:1 and 2:1, respectively, in free-moisture dioxane solvent under an argon atmosphere. Compounds of $\mathrm{ArTeBr}_{3}$ and $\mathrm{Ar}_{2} \mathrm{TeBr}_{2}$ were reduced by the action of ethanolic solution of hydrazine hydrate obtained $\mathrm{Ar}_{2} \mathrm{Te}_{2}$ and $\mathrm{Ar}_{2} \mathrm{Te}$, respectively. Reaction of $\mathrm{Ar}_{2} \mathrm{Te}_{2}$ with excess thionyl chloride or iodine gave the corresponding trihalides $\mathrm{ArTeCl}_{3}$ and $\mathrm{ArTeI}_{3}$, respectively while the reaction of $\mathrm{Ar}_{2} \mathrm{Te}$ with thionyl chloride or iodine gave the corresponding $\mathrm{Ar}_{2} \mathrm{TeCl}_{3}$ and $\mathrm{Ar}_{2} \mathrm{TeI}_{3}$, respectively. The structures were elucidated according to their elemental analysis of carbon, hydrogen and nitrogen (CHN) and some of the spectroscopic techniques such as infrared IR and nuclear magnetic resonance for ${ }^{1} \mathrm{H}$ and ${ }^{13} \mathrm{C}$. The antimicrobial activity for all the synthetic compounds were assayed against both Gram-negative and Gram-positive bacteria by using the agar diffusion method. The tellurated pyrazole derivatives showed a good degree against bacteria growth. In some cases, the antimicrobial activities of the synthetic compounds were better than amoxicillin.

Keywords: pyrazoles; organotellurium; tellurium tetrabromide; transmetalltion reaction; antimicrobial activities

\section{Introduction}

Pyrazoles are one of the important five-membered heterocycles compounds that contain three carbons and two adjacent nitrogen atoms and are considered the most famous class among the azole compounds. In fact, different synthesis procedures have been carried out over the decades $[1,2]$. Generally, the compounds that contain the pyrazole moiety possess many different applications in several fields such as in the fields of pharmaceutical drugs and technology. The literature confirms that pyrazole derivatives are used as antimicrobial, antifungal, antitumor, anti-inflammatory, and antioxidant compounds [3]. A common method for the synthesis of the substituted pyrazoles includes the cyclization reaction of $\alpha, \beta$-unsaturated ketones (chalcones) with hydrazines [4,5].

To the best of our knowledge, through a literature search of SciFinder Scholar and Web of Knowledge, there have been few reported studies related to the synthesis of organotellurium compounds derived from pyrazoles [6,7]. Furthermore, due to the high significant antimicrobial activity of both pyrazoles [2,4] and organic tellurium compounds [8-10], in addition, to our interest in the field of organic synthesis for tellurium compounds by the transmetalation reactions between aryl mercury chlorides 
with tellurium tetrabromide [11,12], in the present work, we synthesized some organic tellurium compounds based on pyrazole and evaluated their microbial activity against four different bacteria.

\section{Results and Discussion}

First, substituted chalcones i-iii and 2-hydrazinyl-3,5-dinitrophenylmercury chloride were prepared, starting according to the procedure in the literature [13]. Organomercuric chlorides containing pyrazole moiety 1-3 were obtained from the corresponding substituted chalcones and 2-hydrazinyl-3,5-dinitrophenylmercury chloride (Scheme 1).<smiles>[R]c1ccc(C(=O)/C=C/c2ccccc2Cl)cc1</smiles><smiles>Cc1cc([N+](=O)[O-])cc([N+](=O)[O-])c1N</smiles>
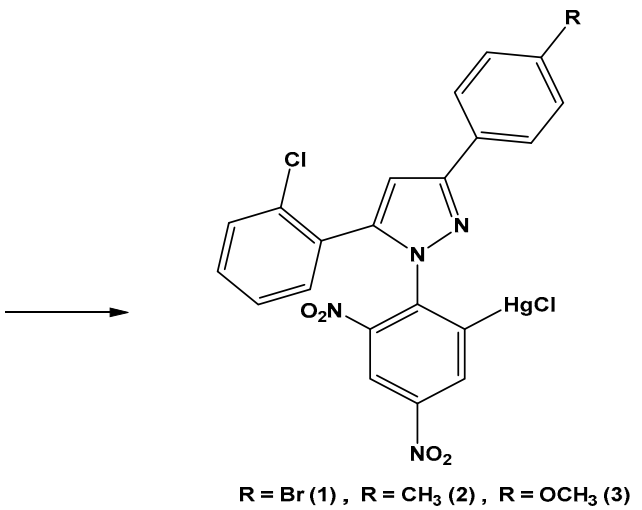

Scheme 1. Preparative methods of compounds 1-3.

Aryltelluium tribromides 4-6 are generated from the reaction of tellurium tetrabromide with $\mathbf{1}$, 2, and 3, respectively, in a 1:1 mole ratio as orange, reddish orange, and yellowish orange solids in moderate yields. In a similar manner, good yields of diaryl tellurium dibromides 7, 8, and 9 were resulted by the reaction of tellurium tetrabromide with $\mathbf{1}, \mathbf{2}$, or 3 , respectively, in a 1:2 mole ratio as orange-yellowish orange solids. Reduction of compounds 4, 5, and 6 by hydrazine hydrate gave the corresponding ditellurides 10, 11, and 12, respectively, in good yields, while the reduction of 7 , 8 , or 9 gave the corresponding tellurides 13, 14, and 15, respectively, as dark red solids in low to moderate yields. The preparative methods of all the new synthesized compounds $\mathbf{4 - 1 5}$ are illustrated in Scheme 2.

Reaction of diaryl ditellurides 10-12 with an excess of thionyl chloride or iodine solutions gave the corresponding aryl tellurium trichlorides $\mathrm{ArTeCl}_{3}$ 16-18, and triiodides $\mathrm{ArTeI}_{3}$ 19-21, respectively (Scheme 3). Reaction of diaryl telluride $\mathrm{Ar}_{2} \mathrm{Te}$ 13-15 with thionyl chloride and iodine solutions gave the corresponding diaryl tellurium dichlorides $\mathrm{Ar}_{2} \mathrm{TeCl}_{2} \mathbf{2 2 - 2 4}$ and diiodides $\mathrm{Ar}_{2} \mathrm{TeI}_{2}$ 25-27, respectively (Scheme 4).

All the synthetic compounds were followed by thin-layer chromatography (TLC) since the reaction of chalcones with aryl hydrazines or reaction of tellurium tetrabromide with aryl mercuric chlorides are critical reactions, so we may expect the possibility of two different compounds (substituted pyrazoles and pyrazolines). However, after purification, no indications of undesired compounds were found upon checking the elemental analysis $\mathrm{CHN}$ and ${ }^{1} \mathrm{H}$ - and ${ }^{13} \mathrm{C}-\mathrm{NMR}$ spectra. Generally, the prepared compounds 1-27 are colored, solid, stable in air, and soluble in DMF and DMSO.

The structures of recrystallized 1-27 were identified by means of spectroscopic techniques and elemental analysis. For the elemental analysis of carbon, hydrogen, and nitrogen of compounds 1-27, both the theoretical (calculated) and practical (found) are approximately identical values. This indicates the validity of the suggested structures. 


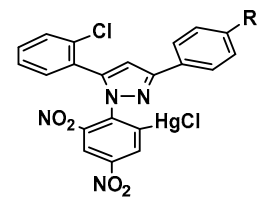

$\mathrm{R}=\mathrm{Br}(1), \mathrm{R}=\mathrm{CH}_{3}(2), \mathrm{R}=\mathrm{OCH}_{3}(3)$
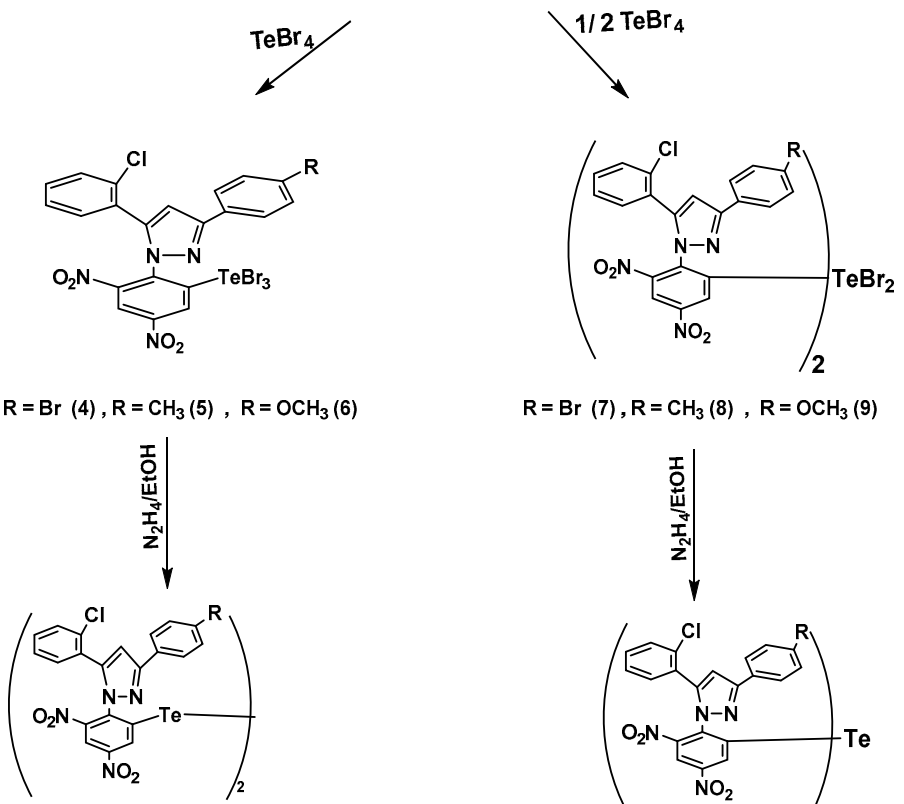

$\mathrm{R}=\mathrm{Br}(7), \mathrm{R}=\mathrm{CH}_{3}(8), \mathrm{R}=\mathrm{OCH}_{3}(9)$

$\mathrm{R}=\mathrm{Br}(10), \mathrm{R}=\mathrm{CH}_{3}(11), \mathrm{R}=\mathrm{OCH}_{3}(12)$

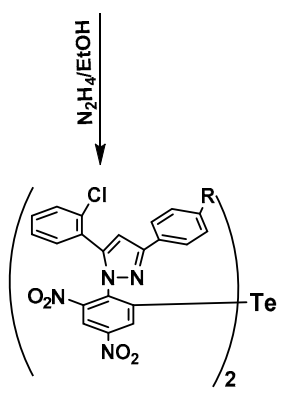

$\mathrm{R}=\mathrm{Br}(13), \mathrm{R}=\mathrm{CH}_{3}(14), \mathrm{R}=\mathrm{OCH}_{3}(15)$

Scheme 2. Preparative methods of compounds 4-15.

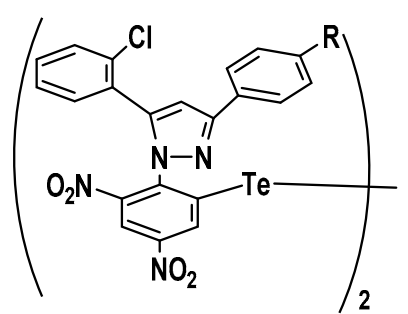

$\mathrm{R}=\mathrm{Br}(10), \mathrm{R}=\mathrm{CH}_{3}(11), \mathrm{R}=\mathrm{OCH}_{3}(12)$

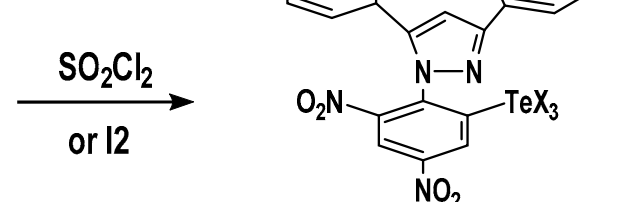

$X=\mathrm{Cl} ; \mathrm{R}=\mathrm{Br}(16), \mathrm{R}=\mathrm{CH}_{3}(17), \mathrm{R}=\mathrm{OCH}_{3}(18)$ $X=I ; R=B r(19), R=C_{3}(20), R=O C_{3}(21)$

Scheme 3. Preparative methods of compounds 16-21.

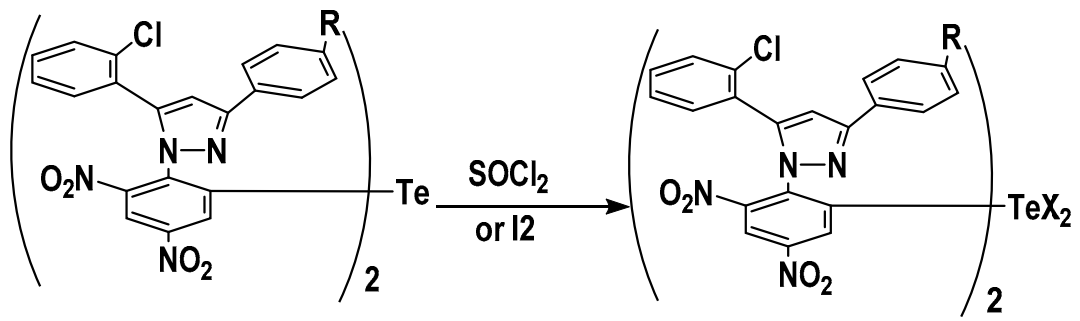

$\mathrm{R}=\mathrm{Br}(13), \mathrm{R}=\mathrm{CH}_{3}(14), \mathrm{R}=\mathrm{OCH}_{3}(15) \quad \mathrm{X}=\mathrm{Cl} ; \mathrm{R}=\mathrm{Br}(22), \mathrm{R}=\mathrm{CH}_{3}(23), \mathrm{R}=\mathrm{OCH}_{3}(24)$

$X=I ; \quad R=B r(25), R=\mathrm{CH}_{3}(26), \mathrm{R}=\mathrm{OCH}_{3}(27)$

Scheme 4. Preparative methods of compounds 22-27. 
The FTIR spectra of all prepared compounds 1-27 showed important vibrational bands in certain regions, especially in the fingerprint (these spectra are available online as supplementary materials Figures S55-S69). The FTIR spectra shows that the disappearance of the stretching band belonged to $\mathrm{C}=\mathrm{O}$ and $\mathrm{C}=\mathrm{C}$ bonds of chalcones in the region between the $1650-1662 \mathrm{~cm}^{-1}$ and $1597-1604 \mathrm{~cm}^{-1}$ regions, respectively, and stretching of $\mathrm{N}-\mathrm{H}$ bond of hydrazine in the $3200-3500 \mathrm{~cm}^{-1}$ region $[4,13]$. On the other hand, the spectra of pyrazole derivatives showed the stretching bands for $\mathrm{C}=\mathrm{N}$ at the 1597-1631 and $C=C$ at $1400-1531 \mathrm{~cm}^{-1}$ regions [4].

${ }^{1} \mathrm{H}$ - and ${ }^{13} \mathrm{C}$ - NMR spectra of 1-27 were carried out in DMSO- $d_{6}$ solutions (these spectra are available online as supplementary materials, Figures S1-S54). These compounds appeared for the expected characteristic signals of all protons in these compounds, which are in good agreement with their suggested molecular formula (Figure 1).

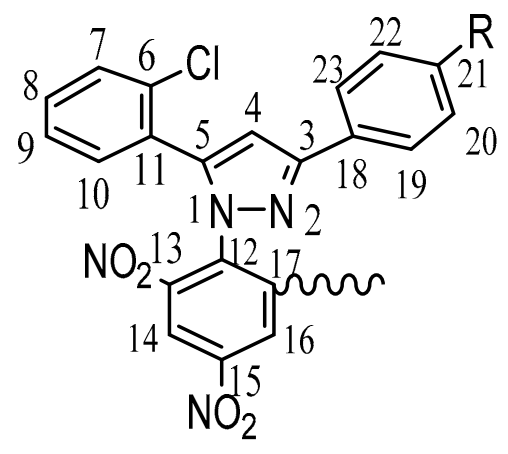

Figure 1. General structure formula.

A singlet signal equivalent to one or two protons at $\delta=5.80-5.96$ ppm was characteristic for $\mathrm{H} 4$ (and $\mathrm{H}^{\prime}$ ) protons. This signal can be considered as evidence of the prepared pyrazole ring $[14,15]$. Multiplet signals at 7.45-8.23 ppm can be assigned to protons of the aromatic rings. For compounds 2, $\mathbf{5}, \mathbf{8}, \mathbf{1 1}, \mathbf{1 4}, \mathbf{1 7}, \mathbf{2 0}, \mathbf{2 3}$, and $\mathbf{2 6}$, the protons of methyl groups that attached on C21(and C21') appeared as a singlet signal at $\delta 2.20-2.22$ ppm. For compounds $3, \mathbf{6}, \mathbf{9}, \mathbf{1 2}, \mathbf{1 5}, \mathbf{1 8}, \mathbf{2 1}, \mathbf{2 4}$, and 27, the spectra showed that the singlet signal in the region $\delta=3.86-3.89$ ppm can be characterized for the $\mathrm{OCH}_{3}$ group at $\mathrm{C} 21$ (and C21') [16].

The signals for the carbon atoms of pyrazole rings can be identified via their corresponding ${ }^{13} \mathrm{C}$ NMR spectra, which were in excellent agreement with previous papers [14,17]. These signals can be considered as more evidence of the correctness of the suggested structures (Table 1). The ${ }^{13} \mathrm{C} \mathrm{NMR}$ spectra of 1-27 revealed a signal at the range 139.50-139.88 ppm due to C3, while the C5 atoms showed a signal at the range of 149.60-149.80 ppm. The ${ }^{13} \mathrm{C}$ NMR spectra of 1-27 appeared as a signal at the region of 103.18-104.66 ppm, which can be assigned to C4. Surprisingly, we found the chemical shift of the C4 atom signal effect from the 3-aryl group, which in turn effects the resonance contributions of the 3-aryl group and the chemical shift difference at C4 of the pyrazole ring. We conclude from the above that the 3-aryl group may be located at the same plane of the pyrazole ring, increasing the resonance contribution of $\pi$-bonds. The ${ }^{13} \mathrm{CNMR}$ spectra of compounds $4-27$ showed that the signal in the 114.90-114.06 ppm region can be identified as carbon atoms that bear the tellurium-carbon bond Te-C. Comparatively, this signal possessed a low chemical shift (high field), which may be due to the polarity of the tellurium-carbon bond. In general, the aromatic carbon signals appear between 124.00-163.43 ppm. The ${ }^{13} \mathrm{C}$ NMR spectra of 2, 5, 8, 11, 14, 17, 20, 23, and 26 showed that the signal within the region 21.59-21.82 ppm was due to the carbon of the methyl group. Compounds 3, 6, 9, $\mathbf{1 2}, \mathbf{1 5}, \mathbf{1 8}, \mathbf{2 1}, \mathbf{2 4}$, and 27 that appeared as a signal in the high field at the range 55.58-56.60 ppm was attributed to the methoxy group. 
Table 1. ${ }^{13} \mathrm{C}$ NMR chemical shifts for compounds 1-15 in DMSO- $d_{6}$.

\begin{tabular}{|c|c|c|c|c|c|c|c|}
\hline \multirow{2}{*}{ Compound } & \multicolumn{3}{|c|}{ Pyrazol Ring } & \multicolumn{2}{|c|}{ Aryl Rings } & \multicolumn{2}{|c|}{ Substituted Groups } \\
\hline & $\mathrm{C} 3$ & $\mathrm{C} 4$ & C5 & Te-C & Ar-H & $\mathrm{CH}_{3}$ & $\mathrm{OCH}_{3}$ \\
\hline 1 & 139.80 & 104.66 & 149.62 & 114.90 & $138.80-124.20$ & & \\
\hline 2 & 139.72 & 104.64 & 149.60 & 114.42 & $132.50-124.00$ & 21.60 & \\
\hline 3 & 139.74 & 104.64 & 149.61 & 114.46 & $163.20-121.15$ & & 56.00 \\
\hline 4 & 139.89 & 104.66 & 149.60 & 114.44 & $138.89-124.28$ & & \\
\hline 5 & 139.70 & 104.40 & 149.80 & 114.00 & $132.41-124.24$ & 21.73 & \\
\hline 6 & 139.77 & 104.43 & 149.82 & 114.06 & $163.41-124.70$ & & 56.60 \\
\hline 7 & 139.60 & 104.66 & 149.66 & 114.88 & $138.91-124.33$ & & \\
\hline 8 & 139.50 & 104.34 & 149.60 & 114.00 & $132.38-124.20$ & 21.80 & \\
\hline 9 & 139.52 & 104.34 & 149.62 & 114.06 & $163.41-124.74$ & & 55.58 \\
\hline 10 & 139.56 & 103.71 & 149.66 & 114.14 & $139.56-124.60$ & & \\
\hline 11 & 139.50 & 103.56 & 149.64 & 114.40 & $133.42-124.77$ & 21.59 & \\
\hline 12 & 139.50 & 103.60 & 149.64 & 114.31 & $163.00-124.00$ & & 55.60 \\
\hline 13 & 139.88 & 103.52 & 149.70 & 114.72 & $138.00-124.10$ & & \\
\hline 14 & 139.84 & 103.18 & 149.65 & 114.60 & $132.41-124.78$ & 21.60 & \\
\hline 15 & 139.86 & 103.43 & 149.65 & 114.65 & $163.43-124.77$ & & 55.59 \\
\hline 16 & 139.88 & 103.24 & 149.78 & 114.70 & $138.00-124.74$ & & \\
\hline 17 & 139.70 & 103.20 & 149.71 & 114.65 & $132.40-124.00$ & 21.50 & \\
\hline 18 & 139.52 & 103.20 & 149.63 & 114.66 & $163.45-124.50$ & & 55.51 \\
\hline 19 & 139.80 & 103.60 & 149.75 & 114.79 & 138..43-124.06 & & \\
\hline 20 & 139.84 & 103.56 & 149.62 & 114.86 & 132.38-125.54 & 21.74 & \\
\hline 21 & 139.77 & 103.45 & 149.64 & 114.75 & $163.03-124.12$ & & 55.59 \\
\hline 22 & 139.84 & 104.50 & 149.75 & 114.91 & 138.09-124.00 & & \\
\hline 23 & 139.80 & 103.35 & 149.60 & 114.64 & $133.00-125.22$ & 21.82 & \\
\hline 24 & 139.63 & 104.34 & 149.66 & 114.80 & $163.00-124.66$ & & 55.60 \\
\hline 25 & 139.85 & 103.95 & 149.79 & 114.83 & $138.05-124.91$ & & \\
\hline 26 & 139.76 & 103.88 & 149.61 & 114.70 & $132.11-124.00$ & 21.77 & \\
\hline 27 & 139.65 & 103.89 & 149.60 & 114.76 & $163.40-124.99$ & & 55.60 \\
\hline
\end{tabular}

The ultra violet-visible for compounds 1-27 showed an absorption peak in the region of 340-500 nm due to $\pi \rightarrow \pi^{*}$ of aromatic rings [18]. Upon investigation of the UV absorptions of the synthetic compounds, it was clearly found that electron-withdrawing $\mathrm{Br}$ on the phenyl ring attached to pyrazole caused a change in the maximum absorption $\left(\lambda_{\max }\right)$ values to longer wavelengths (red shift) while electron-donating groups (Me and $\mathrm{OMe}$ ) moved to shorter wavelengths (blue shift).

It was proven that the molar conductance of organic mercuric compounds 1-3, aryl tellurium trihalides 4-6 and 16-21, and the dihalides 7-9 and 22-27 behaved as for 1:1 electrolytes, which are in good agreement with the previous works in DMSO $[11,12,19,20]$. These observations may be due to the ionic character of $\mathrm{Hg}-\mathrm{Cl}$ and one of the Te-halide bonds in these compounds.

The antimicrobial activity for all pyrazole derivatives 1-27 were assayed against two different types of organisms. The first type was Gram-positive bacteria represented by Staphylococcus aureus ATCC25923 and Candida albicans ATCC2091 and the second was Gram-negative bacteria represented by Escherichia coli ATCC25922 and Pseudomonas aeruginosa ATCC9027. Amoxicillin (10 $\mu \mathrm{g} / \mathrm{disc})$ in DMSO solvent was used as a standard drug by using the agar well diffusion method (Table 2). It can be concluded that all of the compounds (except $\mathrm{ArTeX}_{3} ; \mathrm{X}=\mathrm{Cl}, \mathrm{Br}, \mathrm{I}$ ) showed potent growth inhibition against both Gram-negative and Gram-positive bacteria in different degrees. Rank of the antimicrobial activity of pyrazole derivatives follow the sequence:

$\mathrm{Ar}_{2} \mathrm{Te}$ 13-15 $>\mathrm{Ar}_{2} \mathrm{TeX}_{2}$ 7-9 and 22-27 $>\mathrm{ArHgCl}$ 1-3 $\geq \mathrm{Ar}_{2} \mathrm{Te}_{2}$ 10-12 $>\mathrm{ArTeX}_{3}$ 4-6 and 16-21 
Table 2. Inhibition zones ( $\mathrm{mm}$ ) of synthetic compounds 1-27.

\begin{tabular}{|c|c|c|c|c|}
\hline \multirow{3}{*}{ COMP. } & \multicolumn{4}{|c|}{ Diameter of Inhibition Zone (mm) } \\
\hline & \multicolumn{2}{|c|}{ Gram Positive Bacteria } & \multicolumn{2}{|c|}{ Gram Negative Bacteria } \\
\hline & $\begin{array}{c}\text { Staphylococcus } \\
\text { aureus }\end{array}$ & $\begin{array}{l}\text { Candida } \\
\text { albicans }\end{array}$ & $\begin{array}{c}\text { Escherichia } \\
\text { coli }\end{array}$ & $\begin{array}{c}\text { Pseudomonas } \\
\text { aeruginosa }\end{array}$ \\
\hline 1 & 18 & 16 & 19 & 13 \\
\hline 2 & 14 & 11 & 15 & 10 \\
\hline 3 & 18 & 14 & 16 & 12 \\
\hline 4 & $-*$ & - & - & - \\
\hline 5 & - & - & - & - \\
\hline 6 & - & - & - & 12 \\
\hline 7 & 28 & 25 & 27 & 27 \\
\hline 8 & 21 & 20 & 21 & 23 \\
\hline 9 & 24 & 23 & 25 & 26 \\
\hline 10 & 14 & 17 & 12 & 17 \\
\hline 11 & - & - & 10 & 10 \\
\hline 12 & 10 & 12 & 11 & 11 \\
\hline 13 & 50 & 41 & 42 & 39 \\
\hline 14 & 32 & 30 & 31 & 38 \\
\hline 15 & 34 & 33 & 35 & 37 \\
\hline 16 & - & - & - & - \\
\hline 17 & - & - & - & - \\
\hline 18 & - & - & - & - \\
\hline 19 & - & - & - & - \\
\hline 20 & - & - & - & - \\
\hline 21 & - & - & - & - \\
\hline 22 & 22 & 20 & 21 & 25 \\
\hline 23 & 17 & 18 & 17 & 19 \\
\hline 24 & 21 & 19 & 21 & 22 \\
\hline 25 & 29 & 27 & 22 & 20 \\
\hline 26 & 18 & 18 & 19 & 18 \\
\hline 27 & 21 & 22 & 21 & 19 \\
\hline DMSO & - & 10 & - & - \\
\hline Amoxicillin & 30 & 30 & 30 & 30 \\
\hline
\end{tabular}

*(-) indicates no activity.

In general, the telluride compounds 13, 14, and 15 were more potently active against the bacteria than the other compounds and the control (amoxicillin). This observation may be attributed to the lipophilic properties of the tellurides, which facilitates digestion of the bacteria cellular membrane, or may perhaps be due to their ability to form hydrogen bonds inside the bacteria cell with some active functional groups.

The values of minimum inhibitory concentration (MIC) for pyrazole derivatives that possessed inhibition zones larger than 10 were tested by the agar well diffusion method as shown in Table 3 . The rank of activity was observed as follows: $\mathrm{Br}>\mathrm{OMe}>\mathrm{Me}$. 
Table 3. Minimum inhibition concentration (MIC) mg/ mL of synthetic compounds 1-27.

\begin{tabular}{|c|c|c|c|c|}
\hline \multirow[b]{2}{*}{ Comp. } & \multicolumn{4}{|c|}{ Minimum Inhibition Concentration (MIC) mg/mL } \\
\hline & $\begin{array}{c}\text { Staphylococcus } \\
\text { aureus }\end{array}$ & $\begin{array}{l}\text { Candida } \\
\text { albicans }\end{array}$ & $\begin{array}{c}\text { Escherichia } \\
\text { coli }\end{array}$ & $\begin{array}{c}\text { Pseudomonas } \\
\text { aeruginosa }\end{array}$ \\
\hline 1 & 125 & 250 & 250 & 250 \\
\hline 2 & 500 & - & - & - \\
\hline 3 & 500 & - & 250 & - \\
\hline 4 & $-*$ & - & - & - \\
\hline 5 & - & - & - & - \\
\hline 6 & - & - & - & - \\
\hline 7 & 100 & 250 & 150 & 125 \\
\hline 8 & 125 & 125 & 125 & 100 \\
\hline 9 & 120 & 150 & 100 & 100 \\
\hline 10 & - & 500 & 500 & 250 \\
\hline 11 & - & - & - & 500 \\
\hline 12 & 500 & 500 & 500 & - \\
\hline 13 & 31 & 50 & 63 & 100 \\
\hline 14 & 125 & 200 & 125 & 125 \\
\hline 15 & 50 & 50 & 31 & 50 \\
\hline 16 & - & - & - & - \\
\hline 17 & - & - & - & - \\
\hline 18 & - & - & - & - \\
\hline 19 & - & - & - & - \\
\hline 20 & - & - & - & - \\
\hline 21 & - & - & - & - \\
\hline 22 & 100 & 110 & 120 & 115 \\
\hline 23 & 90 & 90 & 100 & 95 \\
\hline 24 & 95 & 100 & 110 & 100 \\
\hline 25 & 150 & 120 & 125 & 118 \\
\hline 26 & 125 & 110 & 92 & 100 \\
\hline 27 & 130 & 110 & 100 & 105 \\
\hline
\end{tabular}

${ }^{*}(-)$ indicates no activity.

\section{Experimental}

\subsection{Instrumentation}

$\mathrm{CHN}$ analysis was conducted at the University of Al al-Bayt, Al-Mafraq, Jordan by using a Euro vector EA 3000A elemental analysis (Rome, Italy). FTIR spectra for all synthetic compounds were performed by using a FTIR spectrophotometer Shimadzu model 8400 S (Tokyo, Japan) as KBr disk in the range of $4000-400 \mathrm{~cm}^{-1}$ at the University of Basrah. ${ }^{1} \mathrm{H}$ NMR and ${ }^{13} \mathrm{C}$ NMR spectra were measured by using an Ainova $(500 \mathrm{MHz})$ in DMSO- $d_{6}$ solution and tetramethyl silane as the internal standard at Tehran University, Tehran, Islamic Republic of Iran. Ultraviolet-Visible spectra for all synthetic compounds were measured at Basrah University, Basrah, Iraqby using Scan 80D (London, England) in the region $200-800 \mathrm{~nm}$ by using a chloroform solution $1 \times 10^{-4} \mathrm{M}$ and $1 \mathrm{~cm}^{3}$ pathway quartz cells. Measurements of molar conductance were performed for all compounds in DMSO solutions of $1 \times 10^{-3} \mathrm{M}$ at room temperature by using a Konduktoskop model 365B conductivity bridge. Melting points were made by using a Gallenkamp melting point apparatus (London, England). 


\subsection{Synthesis}

\subsubsection{General Method for the Preparation of Aryl Mercury(II) Chlorides}

2-(3-(4-Substitutedphenyl)-5-(2-chlorophenyl)-1H-pyrazol-1-yl)-3,5-dinitrophenyl)mercury(II) chloride 1-3

A mixture of compound 2-hydrazinyl-3,5-dinitrophenylmercury chloride ( $3 \mathrm{mmol})$ in $25 \mathrm{~mL}$ of acetic acid and (4 mmol) of chalcones: 3-(2-chlorophenyl)-1-(4-bromophenyl)-prop-2-en-1-one, 3-(2-chlorophenyl)-1-(4-methylphenyl)prop-2-en-1-one and 3-(2-chlorophenyl)-1-(4-methoxy phenyl)prop-2-en-1-one), respectively, was refluxed for $5 \mathrm{~h}$. Then, a catalytic amount of $\mathrm{HCl}$ (6-8 drops) was added and the mixture was refluxed for $1 \mathrm{~h}$. After cooling, $50 \mathrm{~mL}$ of ice water was added to obtain a yellowish brown solid. The resulting precipitate was filtered, washed several times with water, and recrystallized (twice) from ethanol to obtain yellow solid in $65-77 \%$ yields.

2-(3-(4-Bromophenyl)-5-(2-chlorophenyl)-1H-pyrazol-1-yl)-3,5-dinitrophenyl) mercury (II) chloride (1) Light-yellow crystalline solid; Yield: $77 \%$; M.p.: $193-195^{\circ} \mathrm{C} ; \mathrm{Rf}=0.55$ (ethyl acetate-n-hexane); Molar conductance $\left(\Lambda_{\mathrm{m}}, \mathrm{ohm}^{-1} \mathrm{~cm}^{-1} \mathrm{~mol}^{-1}\right)$ : 35; FTIR $(\mathrm{KBr}) \mathrm{cm}^{-1}$ : FTIR $(\mathrm{KBr}) \mathrm{cm}^{-1}: 3069 \mathrm{w}, 1600 \mathrm{~s}$, $1510 \mathrm{~s}, 1465 \mathrm{~s}, 1438 \mathrm{~s}, 1390 \mathrm{~s}, 1275 \mathrm{~m}, 1211 \mathrm{~s}, 1175 \mathrm{~m}, 1107 \mathrm{~m}, 1065 \mathrm{~m}, 1107 \mathrm{~m}, 1028,976 \mathrm{~m}, 880 \mathrm{~s}, 825 \mathrm{~m}$, $756 \mathrm{~m} ;{ }^{1} \mathrm{H}$ NMR (500 MHz, DMSO- $\left.d_{6}, \delta / \mathrm{ppm}\right): 5.96$ (s, $\left.1 \mathrm{H}, \mathrm{H} 4\right), 7.45-8.23$ (m, 10H, Ar-H); UV-Vis $\left(\lambda_{\max }, \mathrm{nm}\right)$ : 360; Anal. Calculated for $\mathrm{C}_{21} \mathrm{H}_{11} \mathrm{BrCl}_{2} \mathrm{HgN}_{4} \mathrm{O}_{4}$ : C 34.33, H 1.51, N 7.63, Found C 34.40, H 1.51, N 7.69\%.

2-(5-(2-Chlorophenyl)-3-(4-methyl phenyl)-1H-pyrazol-1-yl)-3,5-dinitro phenyl) mercury(II) chloride (2)

Bright-yellow crystalline solid; Yield: 65\%; M.p.: $138-140{ }^{\circ} \mathrm{C}$; Molar conductance $\left(\Lambda_{\mathrm{m}}, \mathrm{ohm}^{-1} \mathrm{~cm}^{-1} \mathrm{~mol}^{-1}\right): 31 ; \mathrm{R}_{\mathrm{f}}=0.68$ (ethyl acetate-n-hexane); FTIR $(\mathrm{KBr}) \mathrm{cm}^{-1}: 3063 \mathrm{w}, 2974 \mathrm{w}$, $2913 \mathrm{w}, 1600 \mathrm{~s}, 1465 \mathrm{~s}, 1438 \mathrm{~s}, 1392 \mathrm{~s}, 1323 \mathrm{~m}, 1273 \mathrm{~s}, 1107 \mathrm{~s}, 1064 \mathrm{~s}, 1030 \mathrm{~m}, 976,821 \mathrm{~m}, 756 \mathrm{~m} ;{ }^{1} \mathrm{H}$ NMR (500 MHz, DMSO- $\left.d_{6}, \delta / \mathrm{ppm}\right):{ }^{1} \mathrm{H}$ NMR (500 MHz, DMSO- $\left.d_{6}, \delta / \mathrm{ppm}\right): 2.20\left(\mathrm{~s}, 3 \mathrm{H}, \mathrm{CH}_{3}\right) ; 5.90(\mathrm{~s}, 1 \mathrm{H}$, $\mathrm{H} 4)$, 7.50-8.20 (m, 10H, Ar-H); UV-Vis $\left(\lambda_{\max }, \mathrm{nm}\right)$ : 350; Anal. Calculated for $\mathrm{C}_{22} \mathrm{H}_{14} \mathrm{Cl}_{2} \mathrm{HgN}_{4} \mathrm{O}_{4}: \mathrm{C}$ 32.98, H 1.76, N 6.99, Found: C 33.02, H 1.80, N 7.01\%.

2-(5-(2-Chlorophenyl)-3-(4-methoxyphenyl)-1H-pyrazol-1-yl)-3,5-dinitrophenyl)mercury(II) chloride (3)

Light-yellow crystalline solid; Yield: 72\%; M.p.: $180-182{ }^{\circ} \mathrm{C}$; Molar conductance $\left(\Lambda_{\mathrm{m}}, \mathrm{ohm}^{-1} \mathrm{~cm}^{-1} \mathrm{~mol}^{-1}\right): 39 ; \mathrm{R}_{\mathrm{f}}=0.51$ (ethyl acetate-n-hexane); FT-IR $(\mathrm{KBr}) \mathrm{cm}^{-1}: 3063 \mathrm{w}, 2974 \mathrm{w}$, 2931 w, 2839 w, 1600 s, 1604 s, 1570 s,1508 s, 1462 s, 1427 s, 1327 s, 1257 s, 1222 m, 1180 s, 1111 s, $1030 \mathrm{~s}, 976 \mathrm{~m}, 825 \mathrm{~m}, 752 \mathrm{~m}, 678 \mathrm{~m} ;{ }^{1} \mathrm{H}$ NMR $\left(500 \mathrm{MHz}, \mathrm{DMSO}-d_{6}, \delta / \mathrm{ppm}\right): 3.83\left(\mathrm{~s}, 6 \mathrm{H}, \mathrm{OCH}_{3}\right)$, $5.81\left(\mathrm{~s}, 2 \mathrm{H}, \mathrm{H} 4\right.$ and $\left.\mathrm{H} 4^{\prime}\right), 7.43-8.22(\mathrm{~m}, 10 \mathrm{H}, \mathrm{Ar}-\mathrm{H})$; UV-Vis $\left(\lambda_{\max }, \mathrm{nm}\right)$ : 348 ; Anal. Calculated for $\mathrm{C}_{22} \mathrm{H}_{14} \mathrm{Cl}_{2} \mathrm{HgN}_{4} \mathrm{O}_{5}: \mathrm{C} 38.41, \mathrm{H} 2.34, \mathrm{~N}$ 8.14, Found: C 38.47, H 2.39, N 8.21\%.

\subsubsection{General Method for the Preparation of Aryl Tellurium Tribromides}

(2-(3-(4-Substitutedphenyl)-5-(2-chlorophenyl)-1H-pyrazol-1-yl)-3,5-dinitrophenyl)tellurium tribromide 4-6

A mixture of tellurium tetrabromide $(1.78 \mathrm{~g}, 4.00 \mathrm{mmol})$ in $35 \mathrm{~mL}$ of dry dioxane and $(4.00 \mathrm{mmol})$ aryl mercuric chlorides $\mathbf{1}, \mathbf{2}$, or 3 , respectively, in $30 \mathrm{~mL}$ of dry dioxane was refluxed with stirring for $6 \mathrm{~h}$ under an argon atmosphere. The resulting solution was filtered hot and on cooling deposited in a 2:1 complex of dioxane and mercuric chloride as white plates, which was filtered off. The filtrate was reduced by a rotary evaporator to give a brown precipitate. Recrystallization of the crude product from a mixture of chloroform and hexane (1:4) gave a yellow crystalline solid in 60-68\% yields.

(2-(3-(4-Bromophenyl)-5-(2-chlorophenyl)-1H-pyrazol-1-yl)-3,5-dinitrophenyl)tellurium tribromide (4)

Light-yellowish-brown crystalline solid; Yield: $68 \%$; M.p.: $212-214{ }^{\circ} \mathrm{C}$; Molar conductance $\left(\Lambda_{\mathrm{m}}, \mathrm{ohm}^{-1} \mathrm{~cm}^{-1} \mathrm{~mol}^{-1}\right): 27 ; \mathrm{R}_{\mathrm{f}}=0.45$ ethyl acetate-n-hexane); FTIR $(\mathrm{KBr}) \mathrm{cm}^{-1}: 3063 \mathrm{w}, 1600 \mathrm{~s}, 1465 \mathrm{~s}$, $1438 \mathrm{~s}, 1392 \mathrm{~s}, 1273 \mathrm{~m}, 1211 \mathrm{~s}, 1172 \mathrm{~m}, 1107 \mathrm{~m}, 1064$ m, 1107 m, 1026, 976 m, 880 s, 821 m, 756 m; 
${ }^{1} \mathrm{H}$ NMR (500 MHz, DMSO- $\left.d_{6}, \delta / \mathrm{ppm}\right): 5.96$ (s, $\left.1 \mathrm{H}, \mathrm{H} 4\right), 7.46-8.20$ (m, 10H, Ar-H); UV-Vis $\left(\lambda_{\max }, \mathrm{nm}\right)$ : 395; Anal. Calcd for $\mathrm{C}_{21} \mathrm{H}_{11} \mathrm{Br}_{4} \mathrm{ClN}_{4} \mathrm{O}_{4} \mathrm{Te}: \mathrm{C} 29.13, \mathrm{H}$ 1.28,N 6.47, Found: C 29.18,H 1.31, N 6.50\%.

(2-(5-(2-Chlorophenyl)-3-(4-methylphenyl)-1H-pyrazol-1-yl)-3,5-dinitrophenyl)tellurium tribromide (5)

Light-yellowish-brown crystalline solid; Yield: $60 \%$; M.p.: $159-161{ }^{\circ} \mathrm{C}$; Molar conductance $\left(\Lambda_{\mathrm{m}}, \mathrm{ohm}^{-1} \mathrm{~cm}^{-1} \mathrm{~mol}^{-1}\right): 29 ; \mathrm{Rf}=0.75$ (ethyl acetate-n-hexane); FTIR $(\mathrm{KBr}) \mathrm{cm}^{-1}: 3075 \mathrm{~m}, 2925 \mathrm{w}$, $1592 \mathrm{~m}, 1463 \mathrm{~m}, 1439 \mathrm{~m}, 1396 \mathrm{~m}, 1325 \mathrm{~s}, 1277 \mathrm{~m}, 1212 \mathrm{~m}, 1172 \mathrm{~m}, 1065 \mathrm{~m}, 1026 \mathrm{~m}, 978 \mathrm{~s}, 820 \mathrm{~m}, 756 \mathrm{~s}$, $710 \mathrm{~m}, 685 \mathrm{~m}$; ${ }^{1} \mathrm{H}$ NMR (500 MHz, DMSO- $\left.d_{6}, \delta / \mathrm{ppm}\right): 2.20\left(\mathrm{~s}, 3 \mathrm{H}, \mathrm{CH}_{3}\right) ; 5.92(\mathrm{~s}, 1 \mathrm{H}, \mathrm{H} 4), 7.45-8.25$ $(\mathrm{m}, 10 \mathrm{H}, \mathrm{Ar}-\mathrm{H})$; UV-Vis $\left(\lambda_{\max }, \mathrm{nm}\right)$ : 382; Anal. Calculated for $\mathrm{C}_{22} \mathrm{H}_{14} \mathrm{Br}_{3} \mathrm{ClN}_{4} \mathrm{O}_{4} \mathrm{Te}: \mathrm{C} 32.98, \mathrm{H} 1.76$, N 6.99, Found: C 33.02, H 1.80, N 7.01\%.

(2-(5-(2-Chlorophenyl)-3-(4-methoxyphenyl)-1H-pyrazol-1-yl)-3,5dinitrophenyl)tellurium tribromide (6)

Light-yellowish brown solid; Yield: $67 \%$; M.p.: $195-197^{\circ} \mathrm{C}$; Molar conductance $\left(\Lambda_{\mathrm{m}}, \mathrm{ohm}^{-1} \mathrm{~cm}^{-1}\right.$ $\mathrm{mol}^{-1}$ ): 26; $\mathrm{R}_{\mathrm{f}}=0.61$ (ethyl acetate- n-hexane); FTIR $(\mathrm{KBr}) \mathrm{cm}^{-1}: 3063 \mathrm{w}, 2904 \mathrm{w}, 2833 \mathrm{w}, 1604 \mathrm{~s}, 1570 \mathrm{~s}$, 1512 m, 1465 m, 1427 s, 1327 s, 1264 m, 1226 m, 1194 m, 1114 m, 1003 s, 914 m, 823 m, 754 m; ${ }^{1}$ H NMR (500 MHz, DMSO- $\left.d_{6}, \delta / \mathrm{ppm}\right): 3.86\left(\mathrm{~s}, 3 \mathrm{H}, \mathrm{OCH}_{3}\right), 5.84(\mathrm{~s}, 1 \mathrm{H}, \mathrm{H} 4), 7.45-8.20(\mathrm{~m}, 10 \mathrm{H}, \mathrm{Ar}-\mathrm{H}) ; \mathrm{UV}-\mathrm{Vis}$ $\left(\lambda_{\max }, \mathrm{nm}\right): 379$; Anal. Calculated for $\mathrm{C}_{22} \mathrm{H}_{14} \mathrm{Br}_{3} \mathrm{ClN}_{4} \mathrm{O}_{4}$ Te: $\mathrm{C} 32.34, \mathrm{H} 1.73, \mathrm{~N}$ 6.86, Found: C 32.30, H 1.78, N 6.99\%.

\subsubsection{General Method for the Preparation of Diaryl Tellurium Dibromides}

Bis[(2-(3-(4-substitutedphenyl)-5-(2-chlorophenyl)-1H-pyrazol-1-yl)-3,5-dinitrophenyl)]tellurium dibromide 7-9

A mixture of tellurium tetrabromide $(0.89 \mathrm{~g}, 2.00 \mathrm{mmol})$ and aryl mercuric chloride 1,2 , or 3 $(4.00 \mathrm{mmol})$ in $35 \mathrm{~mL}$ of dry dioxane was refluxed with stirring for $6 \mathrm{~h}$ under an argon gas atmosphere. The resulting solution was filtered hot and cooled to room temperature. On cooling, a 2:1 complex of dioxane and mercuric halides was separated as white plates and was filtered off immediately. Recrystallization of the product from a mixture of dichloromethane and hexane (1:4) gave an orange-brown to yellowish brown solid in $70-75 \%$ yield.

Bis[(2-(3-(4-bromophenyl)-5-(2-chlorophenyl)-1H-pyrazol-1-yl)-3,5-dinitrophenyl)]tellurium dibromide (7)

Light-orange-brown crystalline solid; Yield: $75 \%$; M.p.: 209-211 ${ }^{\circ} \mathrm{C}$; Molar conductance $\left(\Lambda_{\mathrm{m}}, \mathrm{ohm}^{-1} \mathrm{~cm}^{-1} \mathrm{~mol}^{-1}\right): 32 ; \mathrm{R}_{\mathrm{f}}=0.50$ (ethyl acetate-n-hexane); FTIR $(\mathrm{KBr}) \mathrm{cm}^{-1}: 3059 \mathrm{w}, 1604 \mathrm{~s}$, $1570 \mathrm{~s}, 1516 \mathrm{~s}, 1465 \mathrm{~m}, 1438 \mathrm{~m}, 1338 \mathrm{~s}, 1311 \mathrm{~m}, 1273 \mathrm{~m}, 1211 \mathrm{~s}, 1180 \mathrm{~m}, 1157 \mathrm{~m}, 1041 \mathrm{~s}, 972 \mathrm{~s}, 860 \mathrm{~s}, 790 \mathrm{~m}$, $752 \mathrm{~m}, 717 \mathrm{~m}, 690 \mathrm{~m}, 655 \mathrm{~m}, 578 \mathrm{~m} ;{ }^{1} \mathrm{H}$ NMR ( $\left.500 \mathrm{MHz}, \mathrm{DMSO}-d_{6}, \delta / \mathrm{ppm}\right): 5.95$ (s, 2H, H4 and H4'), 7.46-8.20 (m, 20H, Ar-H); UV-Vis $\left(\lambda_{\max }, \mathrm{nm}\right)$ : 380; Anal. Calculated for $\mathrm{C}_{42} \mathrm{H}_{22} \mathrm{Br}_{4} \mathrm{Cl}_{2} \mathrm{~N}_{8} \mathrm{O}_{8} \mathrm{Te}: \mathrm{C} 39.26$, H 1.73, N 8.72, Found: C 39.30, H 1.75, N 8.74\%.

Bis[(2-(5-(2-Chlorophenyl)-3-(4-methylphenyl)-1H-pyrazol-1-yl)-3,5-dinitrophenyl)]tellurium dibromide (8)

Light-yellowish brown crystalline solid; Yield: $70 \%$; M.p.: $150-152{ }^{\circ} \mathrm{C}$; Molar conductance $\left(\Lambda_{\mathrm{m}}, \mathrm{ohm}^{-1} \mathrm{~cm}^{-1} \mathrm{~mol}^{-1}\right): 25 ; \mathrm{R}_{\mathrm{f}}=0.40$ (ethyl acetate-n-hexane); FTIR $(\mathrm{KBr}) \mathrm{cm}^{-1}: 3063 \mathrm{~m}, 2924 \mathrm{w}$, 1604 s, 1593 m, 1512 m, 1469 m, 1442 m, 1315 s, 1273 m, 1215 m, 1037 m, 1014 m, 976 m, 752 m, 578 m; ${ }^{1} \mathrm{H}$ NMR (500 MHz, DMSO- $\left.d_{6}, \delta / \mathrm{ppm}\right): 2.06\left(\mathrm{~s}, 6 \mathrm{H}, 2 \mathrm{CH}_{3}\right) ; 5.92\left(\mathrm{~s}, 2 \mathrm{H}, \mathrm{H} 4\right.$ and $\left.\mathrm{H} 4{ }^{\prime}\right), 7.45-8.20(\mathrm{~m}, 20 \mathrm{H}$, Ar-H); UV-Vis $\left(\lambda_{\max }, \mathrm{nm}\right)$ : 373; Anal. Calculated for $\mathrm{C}_{44} \mathrm{H}_{28} \mathrm{Br}_{2} \mathrm{Cl}_{2} \mathrm{~N}_{8} \mathrm{O}_{8} \mathrm{Te}$ : C 45.75, H 2.44, N 9.70, Found: C 45.84, H 2.51, N 9.76\%. 
Bis[2-(5-(2-Chlorophenyl)-3-(4-methoxyphenyl)-1H-pyrazol-1-yl)-3,5dinitro phenyl)]tellurium dibromide (9)

Light-yellowish brown crystalline solid; Yield: $71 \%$; M.p.: $191-193{ }^{\circ} \mathrm{C}$; Molar conductance $\left(\Lambda_{\mathrm{m}}, \mathrm{ohm}^{-1} \mathrm{~cm}^{-1} \mathrm{~mol}^{-1}\right)$ : 30; Rf $=0.70$ (ethyl acetate-n-hexane); FTIR $(\mathrm{KBr}) \mathrm{cm}^{-1}: 3063 \mathrm{w}, 2931 \mathrm{w}$, $2850 \mathrm{w}, 1606 \mathrm{~s}, 1571 \mathrm{~s}, 1520 \mathrm{~m}, 1460 \mathrm{~m}, 1430 \mathrm{~m}, 1330 \mathrm{~s}, 1261 \mathrm{~m}, 1227 \mathrm{~m}, 1180 \mathrm{~m}, 1034 \mathrm{~m}, 1014 \mathrm{~m}, 978 \mathrm{~s}$, $830 \mathrm{~m}, 759 \mathrm{~m}, 682 \mathrm{~m}, 578 \mathrm{~m} ;{ }^{1} \mathrm{H}$ NMR (500 MHz, DMSO- $\left.d_{6}, \delta / \mathrm{ppm}\right): 3.86\left(\mathrm{~s}, 6 \mathrm{H}, 2 \mathrm{OCH}_{3}\right), 5.80(\mathrm{~s}, 2 \mathrm{H}$, 2H4), 7.45-8.20 (m, 20H, Ar-H); UV-Vis $\left(\lambda_{\max }, \mathrm{nm}\right)$ : 371; Anal. Calculated for $\mathrm{C}_{44} \mathrm{H}_{28} \mathrm{Br}_{2} \mathrm{Cl}_{2} \mathrm{~N}_{8} \mathrm{O}_{10} \mathrm{Te}$ : C 44.52, H 2.38, N 9.44, Found: C 44.60, H 2.41, N 9.45\%.

\subsubsection{General Method for the Preparation of Diaryl Ditellurides}

Bis[(2-(3-(4-substituted phenyl)-5-(2-chlorophenyl)-1H-pyrazol-1-yl)-3,5-dinitrophenyl)] ditelluride 10-12

Aryl tellurium tribromide $(3.00 \mathrm{mmol})$ was refluxed in ethanol $(25 \mathrm{~mL})$. An ethanolic solution of hydrazine hydrate was added drop by drop to the refluxing solution until the evolution of nitrogen ceased. The resulting solution was cooled to room temperature and poured into $100 \mathrm{~mL}$ of distilled water and extract with diethyl ether $(4 \times 30 \mathrm{~mL})$. The etheric extracts were dried over an anhydrous calcium chloride. Evaporation of solvent afforded a dark red solid of compounds. The resulting precipitate was recrystallized by ethanol and gave a dark red solid in $61-68 \%$ yields.

Bis[(2-(3-(4-bromophenyl)-5-(2-chlorophenyl)-1H-pyrazol-1-yl)-3,5-dinitrophenyl)] ditelluride (10)

Dark red crystalline solid; Yield: $68 \%$; M.p.: $100-102{ }^{\circ} \mathrm{C}$; Molar conductance $\left(\Lambda_{\mathrm{m}}, \mathrm{ohm}^{-1} \mathrm{~cm}^{-1}\right.$ $\mathrm{mol}^{-1}$ ): 9; $\mathrm{R}_{\mathrm{f}}=0.62$ (ethyl acetate-n-hexane); FTIR $(\mathrm{KBr}) \mathrm{cm}^{-1}: 3072 \mathrm{w}, 2928 \mathrm{w}, 28431 \mathrm{w}, 1600 \mathrm{~s}, 1570 \mathrm{~s}$, $1520 \mathrm{~m}, 1465 \mathrm{~m}, 1431 \mathrm{~m}, 1331 \mathrm{~s}, 1266 \mathrm{~m}, 1226 \mathrm{~m}, 1181 \mathrm{~m}, 1033 \mathrm{~m}, 1010 \mathrm{~m}, 976 \mathrm{~s}, 829 \mathrm{~m}, 758 \mathrm{~m}, 684 \mathrm{~m}$, $578 \mathrm{~m} ;{ }^{1} \mathrm{H}$ NMR (500 MHz, DMSO- $\left.d_{6}, \delta / \mathrm{ppm}\right): 5.96$ (s, 2H, H4, and H4'), 7.47-8.23 (m, 20H, Ar-H); UV-Vis $\left(\lambda_{\max }, \mathrm{nm}\right): 500$; Anal. Calculated for $\mathrm{C}_{42} \mathrm{H}_{22} \mathrm{Br}_{2} \mathrm{Cl}_{2} \mathrm{~N}_{8} \mathrm{O}_{8} \mathrm{Te}_{2}: \mathrm{C} 40.27, \mathrm{H} 1.77, \mathrm{~N}$ 8.95, Found: C $40.33, \mathrm{H} 1.80, \mathrm{~N} 9.00 \%$.

Bis[(2-(5-(2-Chlorophenyl)-3-(4-methylphenyl)-1H-pyrazol-1-yl)-3,5-dinitrophenyl)] ditelluride (11)

Dark red crystalline solid; Yield: $61 \%$; M.p.: $91-93{ }^{\circ} \mathrm{C}$; Molar conductance $\left(\Lambda_{\mathrm{m}}, \mathrm{ohm}^{-1} \mathrm{~cm}^{-1}\right.$ $\mathrm{mol}^{-1}$ ): 11; $\mathrm{R}_{\mathrm{f}}=0.68$ (ethyl acetate-n-hexane); FTIR $(\mathrm{KBr}) \mathrm{cm}^{-1}: 3063 \mathrm{w}, 2924 \mathrm{w}, 1631 \mathrm{~s}, 1569 \mathrm{~m}, 1539 \mathrm{~m}$, $1481 \mathrm{~m}, 1455 \mathrm{~m}, 1399 \mathrm{~m}, 1335 \mathrm{~s}, 1269 \mathrm{~s}, 1211 \mathrm{~m}, 1134 \mathrm{~m}, 1068 \mathrm{~m}, 1045 \mathrm{~m}, 852 \mathrm{~m}, 826 \mathrm{~m}, 768 \mathrm{~m}, 628 \mathrm{w}$; ${ }^{1} \mathrm{H}$ NMR (500 MHz, DMSO- $\left.d_{6}, \delta / \mathrm{ppm}\right): 2.33\left(\mathrm{~s}, 6 \mathrm{H}, 2 \mathrm{CH}_{3}\right) ; 5.91\left(\mathrm{~s}, 2 \mathrm{H}, \mathrm{H} 4\right.$ and $\left.\mathrm{H}^{\prime}\right), 7.42-8.20(\mathrm{~m}, 20 \mathrm{H}$, Ar-H); UV-Vis $\left(\lambda_{\max }, \mathrm{nm}\right)$ : 491; Anal. Calculated for $\mathrm{C}_{44} \mathrm{H}_{28} \mathrm{Cl}_{2} \mathrm{~N}_{8} \mathrm{O}_{8} \mathrm{Te}_{2}$ : C 47.07, H 2.51, N 9.98, Found: C 47.10, H 2.50, N 10.10\%.

Bis[(2-(5-(2-Chlorophenyl)-3-(4-methoxyphenyl)-1H-pyrazol-1-yl)-3,5dinitro phenyl)] ditelluride (12)

Dark red crystalline solid; Yield: $62 \%$; M.p.: $99-101{ }^{\circ} \mathrm{C}$; Molar conductance $\left(\Lambda_{\mathrm{m}}, \mathrm{ohm}^{-1} \mathrm{~cm}^{-1}\right.$ $\mathrm{mol}^{-1}$ ): 10; $\mathrm{R}_{\mathrm{f}}=0.55$ (ethyl acetate- $\mathrm{n}$-hexane); $\mathrm{IR}(\mathrm{KBr}) \mathrm{cm}^{-1}: 3063 \mathrm{w}, 2974 \mathrm{w}, 2931 \mathrm{w}, 2839 \mathrm{w}, 1604 \mathrm{~m}$, 1570 s, 1512 m, 1485 m, 1427 m, 1327 m, 1261 s, 1226 m, 1184 m, 1033 s, 1014 m, 976 m, 825 m, 756 m, $682 \mathrm{~m}, 578 \mathrm{~m} ;{ }^{1} \mathrm{H}$ NMR $\left(500 \mathrm{MHz}, \mathrm{DMSO}-d_{6}, \delta / \mathrm{ppm}\right) 3.86\left(\mathrm{~s}, 6 \mathrm{H}, 2 \mathrm{OCH}_{3}\right), 5.80(\mathrm{~s}, 2 \mathrm{H}, 2 \mathrm{H} 4), 7.45-8.20$ (m, 20H, Ar-H); UV-Vis $\left(\lambda_{\max }, \mathrm{nm}\right)$ : 490; Anal. Calculated for $\mathrm{C}_{44} \mathrm{H}_{28} \mathrm{Cl}_{2} \mathrm{~N}_{8} \mathrm{O}_{10} \mathrm{Te}_{2}$ : C 45.70, H 2.35, N 9.68, Found: C 45.76, H 2.44, N 9.70\%.

\subsubsection{General Method for the Preparation of Diaryl Tellurides}

Bis[(2-(3-(4-substituted phenyl)-5-(2-chlorophenyl)-1H-pyrazol-1-yl)-3,5-dinitrophenyl)] telluride 13-15

Diaryl tellurium dibromides (i.e., compounds 7, 8, or 9) $(2.00 \mathrm{mmol})$ was dissolved in $25 \mathrm{~mL}$ of ethanol and refluxed. A solution of hydrazine hydrate in ethanol was added drop wisely to the refluxed solution until nitrogen evaluation ceased. The resulting solution was poured into $500 \mathrm{~mL}$ of distilled ice water to afford a yellow solid. The crude product was twice recrystallized from a mixture of ethanol and dichloromethane to obtain a yellow or yellowish brown solid in 58-67\% yields. 
Bis[(2-(3-(4-bromophenyl)-5-(2-chlorophenyl)-1H-pyrazol-1-yl)-3,5-dinitrophenyl)] telluride (13)

Light-yellowish brown crystalline solid; Yield: 67\%; M.p.: $88-90{ }^{\circ} \mathrm{C}$; Molar conductance $\left(\Lambda_{\mathrm{m}}, \mathrm{ohm}^{-1} \mathrm{~cm}^{-1} \mathrm{~mol}^{-1}\right): 13 ; \mathrm{R}_{\mathrm{f}}=0.73$ (ethyl acetate-n-hexane); FTIR $(\mathrm{KBr}) \mathrm{cm}^{-1}: 3063 \mathrm{w}, 1609 \mathrm{~s}, 1597$, $1465 \mathrm{~m}, 1438 \mathrm{~m}, 1323 \mathrm{~s}, 1273 \mathrm{~m}, 1311 \mathrm{~m}, 1272 \mathrm{~m}, 1211 \mathrm{~s}, 1181 \mathrm{~m}, 1157 \mathrm{~m}, 1041 \mathrm{~s}, 986 \mathrm{~s}, 860 \mathrm{~s}, 790 \mathrm{~m}$, $756 \mathrm{~m}, 719 \mathrm{~m}, 667 \mathrm{~m}, 655 \mathrm{~m}, 578 \mathrm{~m} ;{ }^{1} \mathrm{H}$ NMR (500 MHz, DMSO- $d_{6}, \delta / \mathrm{ppm}$ ): 5.94 (s, 2H, H4 and H4'), 7.44-8.23 (m, 20H, Ar-H); UV-Vis $\left(\lambda_{\max }, \mathrm{nm}\right)$ : 358; Anal. Calculated for $\mathrm{C}_{21} \mathrm{H}_{11} \mathrm{BrCl}_{3} \mathrm{~N}_{4} \mathrm{O}_{4}$ Te: $\mathrm{C} 34.43$, H 1.51, N 7.65, Found: C 34.50, H 1.51, N7.71\%.

Bis[(2-(5-(2-Chlorophenyl)-3-(4-methylphenyl)-1H-pyrazol-1-yl)-3,5-dinitrophenyl)] telluride (14)

Light-yellow crystalline solid; Yield: 58\%; M.p.: $80-82{ }^{\circ} \mathrm{C}$; Molar conductance $\left(\Lambda_{\mathrm{m}}, \mathrm{ohm}^{-1} \mathrm{~cm}^{-1} \mathrm{~mol}^{-1}\right): 8 ; \mathrm{R}_{\mathrm{f}}=0.65$ (ethyl acetate-n-hexane); FTIR $(\mathrm{KBr}) \mathrm{cm}^{-1}: 3059 \mathrm{w}, 1600 \mathrm{~s}$, $1567 \mathrm{~s}, 1513 \mathrm{~s}, 1460 \mathrm{~m}, 1440 \mathrm{~m}, 1332 \mathrm{~s}, 1310 \mathrm{~m}, 1270 \mathrm{~m}, 1210 \mathrm{~s}, 1181 \mathrm{~m}, 1154 \mathrm{~m}, 1040 \mathrm{~s}, 970 \mathrm{~s}, 861 \mathrm{~s}$, $788 \mathrm{~m}, 751 \mathrm{~m}, 719 \mathrm{~m}, 691 \mathrm{~m}, 657 \mathrm{~m}, 573 \mathrm{~m} ;{ }^{1} \mathrm{H}$ NMR (500 MHz, DMSO-d $\left.d_{6}, \delta / \mathrm{ppm}\right): 2.06$ (s, 6H, 2CH ); $^{2}$ $5.90\left(\mathrm{~s}, 2 \mathrm{H}, \mathrm{H} 4\right.$ and $\left.\mathrm{H} 4^{\prime}\right), 7.46-8.20(\mathrm{~m}, 20 \mathrm{H}, \mathrm{Ar}-\mathrm{H})$; UV-Vis $(\lambda \max , \mathrm{nm})$ : 345; Anal. Calculated for $\mathrm{C}_{44} \mathrm{H}_{28} \mathrm{Cl}_{2} \mathrm{~N}_{8} \mathrm{O}_{8}$ Te: C 53.10, H 2.84, N 11.26, Found: C 53.13, H 2.90, N 11.33\%.

Bis[(2-(5-(2-Chlorophenyl)-3-(4-methoxyphenyl)-1H-pyrazol-1-yl)-3,5-dinitrophenyl)] telluride (15)

Light-yellow crystalline; Yield: $65 \%$; M.p.: $87-85^{\circ} \mathrm{C}$; Molar conductance $\left(\Lambda_{\mathrm{m}}, \mathrm{ohm}^{-1} \mathrm{~cm}^{-1} \mathrm{~mol}^{-1}\right)$ : 11; $R_{\mathrm{f}}=0.60$ (ethyl acetate-n-hexane); FTIR $(\mathrm{KBr}) \mathrm{cm}^{-1}: 3063 \mathrm{w}, 2974 \mathrm{w}, 2931 \mathrm{w}, 2839 \mathrm{w}, 1604 \mathrm{~m}, 1570 \mathrm{~s}$, $1512 \mathrm{~s}, 1465 \mathrm{~m}, 1427 \mathrm{~m}, 1327 \mathrm{~s}, 1261 \mathrm{~m}, 1226 \mathrm{~m}, 1180 \mathrm{~m}, 1111 \mathrm{~m}, 1033 \mathrm{~m}, 1014 \mathrm{~m}, 976 \mathrm{~s}, 825 \mathrm{~m}, 756 \mathrm{~m}$, $682 \mathrm{~m}, 578 \mathrm{~m}, 505 \mathrm{~m} ;{ }^{1} \mathrm{H}$ NMR (500 MHz, DMSO- $\left.d_{6}, \delta / \mathrm{ppm}\right): 3.83\left(\mathrm{~s}, 6 \mathrm{H}, 2 \mathrm{OCH}_{3}\right), 5.81(\mathrm{~s}, 2 \mathrm{H}, \mathrm{H} 4$, and $\left.\mathrm{H}^{\prime}\right), 7.43-8.22(\mathrm{~m}, 20 \mathrm{H}, \mathrm{Ar}-\mathrm{H})$; UV-Vis $\left(\lambda_{\max }, \mathrm{nm}\right)$ : 341 ; Anal. Calculated for $\mathrm{C}_{44} \mathrm{H}_{28} \mathrm{Cl}_{2} \mathrm{~N}_{8} \mathrm{O}_{10} \mathrm{Te}$ : C 51.45, H 2.75, N 10.91, Found: C 51.19, H 2.82, N 11.01\%.

\subsubsection{General Method for the Preparation of Diaryl Tellurium Trichlorides}

(2-(3-(4-substituedphenyl)-5-(2-chlorophenyl)-1H-pyrazol-1-yl)-3,5-dinitrophenyl)tellurium trichloride 16-21

Thionyl chloride $(0.12 \mathrm{~g}, 1.00 \mathrm{mmol})$ in $15 \mathrm{~mL}$ of ethanol was added drop wisely to an ethanolic solution of diaryl ditellurides compounds (i.e., compounds 10,11 or 12) $(1.00 \mathrm{mmol})$ with stirring at room temperature for 30 minutes. A yellow precipitate was formed immediately. Recrystallization by ethanol gave an yellow solid of compounds 16-18.

(2-(3-(4-Bromophenyl)-5-(2-chlorophenyl)-1H-pyrazol-1-yl)-3,5-dinitrophenyl)tellurium trichloride (16)

Yellow brown crystalline solid; 82 Yield: \%; M.p.: $168-170{ }^{\circ} \mathrm{C}$; Molar conductance $\left(\Lambda_{\mathrm{m}}, \mathrm{ohm}^{-1} \mathrm{~cm}^{-1} \mathrm{~mol}^{-1}\right): 33 ; \mathrm{R}_{\mathrm{f}}$ (ethyl acetate- n-hexane) $=0.81 ; \mathrm{FT}-\mathrm{IR}(\mathrm{KBr}) \mathrm{cm}^{-1}: 3059 \mathrm{w}, 1604 \mathrm{~s}$, $1570 \mathrm{~s}, 1465 \mathrm{~s}, 1438 \mathrm{~s}, 1138 \mathrm{~s}, 1311 \mathrm{~s}, 1273 \mathrm{~s}, 1211 \mathrm{~s}, 1180 \mathrm{~s}, 1157 \mathrm{~s}, 1041 \mathrm{~m}, 972 \mathrm{~s}, 850 \mathrm{~m}, 790 \mathrm{~m}, 717 \mathrm{~m}$, $748 \mathrm{~s}, 717 \mathrm{~m}, 690 \mathrm{~m}, 659 \mathrm{~m} ;{ }^{1} \mathrm{H}$ NMR (500 MHz, DMSO- $d_{6}, \delta / \mathrm{ppm}$ ): 5.95 (s, 2H, H4 and H4'), 7.45-8.20 (m, 20H, Ar-H); UV-Vis $\left(\lambda_{\max }, \mathrm{nm}\right)$ : 400; Anal. Calculated for $\mathrm{C}_{21} \mathrm{H}_{11} \mathrm{BrCl}_{4} \mathrm{~N}_{4} \mathrm{O}_{4}$ Te: $\mathrm{C} 51.45, \mathrm{H} 2.75$, N 10.91, Found: C 51.19, H 2.82, N 11.01\%.

(2-(3-(4-Methylphenyl)-5-(2-chlorophenyl)-1H-pyrazol-1-yl)-3,5-dinitrophenyl)tellurium trichloride (17)

Yellow crystalline solid; 76 Yield: \%; M.p.: $160-162{ }^{\circ} \mathrm{C}$; Molar conductance $\left(\Lambda_{\mathrm{m}}, \mathrm{ohm}^{-1} \mathrm{~cm}^{-1}\right.$ $\mathrm{mol}^{-1}$ ): 30; $\mathrm{R}_{\mathrm{f}}$ (ethyl acetate- n-hexane) $=0.75 ;$ FTIR $(\mathrm{KBr}) \mathrm{cm}^{-1}$ : FT-IR $(\mathrm{KBr}) \mathrm{cm}^{-1}: 3065 \mathrm{~m}, 2930 \mathrm{w}$, $1631 \mathrm{~m}, 1590 \mathrm{~m}, 1463 \mathrm{~m}, 1433 \mathrm{~m}, 1390 \mathrm{~m}, 1327 \mathrm{~s}, 1275 \mathrm{~m}, 1216 \mathrm{~m}, 1170 \mathrm{~m}, 1066 \mathrm{~m}, 1022 \mathrm{~m}, 971 \mathrm{~s}, 821 \mathrm{~m}$, $756 \mathrm{~s}, 710 \mathrm{~m}, 687 \mathrm{~m} ;{ }^{1} \mathrm{H}$ NMR (500 MHz, DMSO- $\left.d_{6}, \delta / \mathrm{ppm}\right): 2.20\left(\mathrm{~s}, 6 \mathrm{H}, 2 \mathrm{CH}_{3}\right), 5.90\left(\mathrm{~s}, 2 \mathrm{H}, \mathrm{H} 4\right.$ and $\left.\mathrm{H} 4^{\prime}\right)$, 7.45-8.20 (m, 20H, Ar-H); UV-Vis $\left(\lambda_{\max }, \mathrm{nm}\right)$ : 375; Anal. Calculated for $\mathrm{C}_{22} \mathrm{H}_{14} \mathrm{Cl}_{4} \mathrm{~N}_{4} \mathrm{O}_{4} \mathrm{Te}: \mathrm{C} 38.64$, H 2.06, N 8.19, Found: C 38.70, H 2.00, N 8.24\%. 
(2-(3-(4-Methoxyphenyl)-5-(2-chlorophenyl)-1H-pyrazol-1-yl)-3,5-dinitrophenyl)tellurium trichloride (18)

Yellow crystalline solid; 79 Yield: \%; M.p.: $155-156^{\circ} \mathrm{C}$; Molar conductance $\left(\Lambda_{\mathrm{m}}, \mathrm{ohm}^{-1} \mathrm{~cm}^{-1}\right.$ $\left.\mathrm{mol}^{-1}\right): 24 ; \mathrm{R}_{\mathrm{f}}\left(\right.$ ethyl acetate- n-hexane) $=0.70 ; \operatorname{FTIR}(\mathrm{KBr}) \mathrm{cm}^{-1}: 3060 \mathrm{w}, 2911 \mathrm{w}, 2835 \mathrm{w}, 1600 \mathrm{~s}, 1568 \mathrm{~s}$, $1511 \mathrm{~m}, 1462 \mathrm{~m}, 1425 \mathrm{~s}, 1324 \mathrm{~s}, 1265 \mathrm{~m}, 1230 \mathrm{~m}, 1195 \mathrm{~m}, 1112 \mathrm{~m}, 1005 \mathrm{~s}, 914 \mathrm{~m}, 825 \mathrm{~m}, 759 \mathrm{~m} ;{ }^{1} \mathrm{H}$ NMR $\left(500 \mathrm{MHz}, \mathrm{DMSO}-\mathrm{d}_{6}, \delta / \mathrm{ppm}\right):{ }^{1} \mathrm{H}$ NMR $\left(500 \mathrm{MHz}, \mathrm{DMSO}-d_{6}, \delta / \mathrm{ppm}\right): 3.86\left(\mathrm{~s}, 3 \mathrm{H}, \mathrm{OCH}_{3}\right), 5.82(\mathrm{~s}, 1 \mathrm{H}$, $\mathrm{H} 4), 7.45-8.22(\mathrm{~m}, 10 \mathrm{H}, \mathrm{Ar}-\mathrm{H})$; UV-Vis $\left(\lambda_{\max }, \mathrm{nm}\right)$ : 370; Anal. Calculated for $\mathrm{C}_{22} \mathrm{H}_{14} \mathrm{Cl}_{4} \mathrm{~N}_{4} \mathrm{O}_{4} \mathrm{Te}$ : C 51.45, H 2.75, N 10.91, Found: C 51.19, H 2.82, N 11.01\%.

\subsubsection{General Method for the Preparation of Diaryl Tellurium Triiodides}

(2-(3-(4-Substitutedphenyl)-5-(2-chlorophenyl)-1H-pyrazol-1-yl)-3,5-dinitrophenyl)tellurium triiodide 19-21

A solution of iodine $(0.10 \mathrm{~g}, 0.78 \mathrm{mmol})$ in $10 \mathrm{~mL}$ of ethanol added to a solution of diaryl ditelluride compounds 10, 11, or $12(0.78 \mathrm{mmol})$ in $20 \mathrm{~mL}$ ethanol with stirring at room temperature for $30 \mathrm{~min}$ gave a brown solid of compounds 19,20 , and 21, respectively.

(2-(3-(4-Bromophenyl)-5-(2-chlorophenyl)-1H-pyrazol-1-yl)-3,5-dinitrophenyl)tellurium triiodide (19) Yellowish brown crystalline solid; 73 Yield: \%; M.p.: $147-159{ }^{\circ} \mathrm{C}$; Molar conductance $\left(\Lambda_{\mathrm{m}}, \mathrm{ohm}^{-1} \mathrm{~cm}^{-1} \mathrm{~mol}^{-1}\right)$ : 30; $\mathrm{R}_{\mathrm{f}}$ (ethyl acetate-n-hexane) $=0.45 ;$ FTIR $(\mathrm{KBr}) \mathrm{cm}^{-1}$ : FTIR $(\mathrm{KBr})$ $\mathrm{cm}^{-1}: 3055 \mathrm{w}, 1591 \mathrm{~s}, 1460 \mathrm{~s}, 1439 \mathrm{~s}, 1391 \mathrm{~s}, 1268 \mathrm{~m}, 1214 \mathrm{~s}, 1177 \mathrm{~m}, 1100 \mathrm{~m}, 1065 \mathrm{~m}, 1105 \mathrm{~m}, 1025$, $971 \mathrm{~m}, 882 \mathrm{~s}, 820 \mathrm{~m}, 756 \mathrm{~m} ;{ }^{1} \mathrm{H}$ NMR (500 MHz, DMSO- $\left.d_{6}, \delta / \mathrm{ppm}\right): 5.96$ (s, 2H, H4, and H4'), 7.45-8.22 $(\mathrm{m}, 20 \mathrm{H}, \mathrm{Ar}-\mathrm{H})$; $\mathrm{UV}-\mathrm{Vis}\left(\lambda_{\max }, \mathrm{nm}\right): 390$; Anal. Calculated for $\mathrm{C}_{21} \mathrm{H}_{11} \mathrm{I}_{3} \mathrm{BrClN}_{4} \mathrm{O}_{4} \mathrm{Te}: \mathrm{C} 25.05, \mathrm{H}$ 1.10, N 5.56, Found: C 25.09, H 1.17, N 5.63.01\%.

(2-(3-(4-Methylphenyl)-5-(2-chlorophenyl)-1H-pyrazol-1-yl)-3,5-dinitrophenyl)tellurium triiodide (20) Yellow crystalline solid; 76 Yield: \%; M.p.: $122-120^{\circ} \mathrm{C}$; Molar conductance $\left(\Lambda_{\mathrm{m}}, \mathrm{ohm}^{-1} \mathrm{~cm}^{-1} \mathrm{~mol}^{-1}\right)$ : 29; $\mathrm{R}_{\mathrm{f}}$ (ethyl acetate-n-hexane) $=0.61$; FTIR $(\mathrm{KBr}) \mathrm{cm}^{-1}: 3063 \mathrm{~m}, 2924 \mathrm{w}, 1597 \mathrm{~m}, 1465 \mathrm{~m}, 1438 \mathrm{~m}, 1392 \mathrm{~m}$, 1323 s, 1273 m, 1211 m, 1172 m, 1064 m, 1026 m, 978 s, 821 m, 756 s, 709 m, 687 m; ${ }^{1} \mathrm{H}$ NMR (500 MHz, DMSO- $\left.d_{6}, \delta / \mathrm{ppm}\right): 2.22\left(\mathrm{~s}, 3 \mathrm{H}, \mathrm{CH}_{3}\right) ; 5.90(\mathrm{~s}, 1 \mathrm{H}, \mathrm{H} 4), 7.46-8.20(\mathrm{~m}, 10 \mathrm{H}, \mathrm{Ar}-\mathrm{H})$; UV-Vis $\left(\lambda_{\max }, \mathrm{nm}\right): 395$; Anal. Calculated for $\mathrm{C}_{22} \mathrm{H}_{14} \mathrm{I}_{3} \mathrm{ClN}_{4} \mathrm{O}_{4}$ Te: C 27.58, H 1.50, N 5.95, Found: C 28.10, H 1.51, N 6.01\%.

(2-(3-(4-Methoxyphenyl)-5-(2-chlorophenyl)-1H-pyrazol-1-yl)-3,5-dinitrophenyl)tellurium triiodide (21)

Yellowish brown crystalline solid; 70 Yield: \%; M.p.: $113-105{ }^{\circ} \mathrm{C}$; Molar conductance $\left(\Lambda_{\mathrm{m}}, \mathrm{ohm}^{-1} \mathrm{~cm}^{-1} \mathrm{~mol}^{-1}\right): 22 ; R_{\mathrm{f}}($ ethyl acetate-n-hexane $)=65$; FTIR $(\mathrm{KBr}) \mathrm{cm}^{-1}: 3059 \mathrm{w}, 2905 \mathrm{w}, 2835 \mathrm{w}$, 1598 s, 1570 s, 1515 m, 1465 m, 1428 s, 1327 s, 1265 m, 1227 m, 1192 m, 1117 m, 1013 s, 915 m, 820 m, 755 m; ${ }^{1} \mathrm{H}$ NMR $\left(500 \mathrm{MHz}, \mathrm{DMSO}-d_{6}, \delta / \mathrm{ppm}\right): 3.8\left(\mathrm{~s}, 6 \mathrm{H}, 2 \mathrm{OCH}_{3}\right), 5.81\left(\mathrm{~s}, 2 \mathrm{H}, \mathrm{H} 4\right.$, and $\left.\mathrm{H} 4^{\prime}\right), 7.43-8.22(\mathrm{~m}, 20 \mathrm{H}$, Ar-H); UV-Vis $\left(\lambda_{\max }, \mathrm{nm}\right)$ : 391; Anal. Calculated for $\mathrm{C}_{22} \mathrm{H}_{14} \mathrm{I}_{3} \mathrm{ClN}_{4} \mathrm{O}_{4}$ Te: C 27.58, H 1.47, N 5.85, Found: C 27.62, H 1.41, N 6.94\%.

\subsubsection{General Method for the Preparation of Diaryl Tellurium Dichlorides}

Bis[(2-(3-(4-substitutedphenyl)-5-(2-chlorophenyl)-1H-pyrazol-1-yl)-3,5-dinitrophenyl)]tellurium dichlorides 22-24

Thionyl chloride $(0.12 \mathrm{~g}, 1.00 \mathrm{mmol})$ in $15 \mathrm{~mL}$ of ethanol was added drop wise to an ethanolic solution of diaryl tellurides compounds (i.e., compounds 13, 14, or 15) (1.00 mmol) with stirring at room temperature for 30 minutes. A yellow precipitate was formed immediately. Recrystallization by ethanol gave a yellow solid of compounds 22-24.

Bis[(2-(3-(4-bromophenyl)-5-(2-chlorophenyl)-1H-pyrazol-1-yl)-3,5-dinitrophenyl)]tellurium dichloride (22)

Yellowish orange crystalline solid; Yield: $83 \%$; M.p.: $187-180{ }^{\circ} \mathrm{C}$; Molar conductance $\left(\Lambda_{\mathrm{m}}, \mathrm{ohm}^{-1} \mathrm{~cm}^{-1} \mathrm{~mol}^{-1}\right): 20 ; R_{\mathrm{f}}=0.60$ (ethyl acetate-n-hexane); FTIR $(\mathrm{KBr}) \mathrm{cm}^{-1}: 3063 \mathrm{w}, 1660 \mathrm{~s}, 1609 \mathrm{~s}$, 
1597, 1465 m, 1438 m, 1323 s, 1273 m, 1311 m, 1272 m, 1211 s, 1181 m, 1157 m, 1041 s, 986 s, 860 s, 790 m, $756 \mathrm{~m}, 719 \mathrm{~m}, 667 \mathrm{~m}, 655 \mathrm{~m}, 578 \mathrm{~m} ;{ }^{1} \mathrm{H}$ NMR $\left(500 \mathrm{MHz}, \mathrm{DMSO}-d_{6}, \delta / \mathrm{ppm}\right): 5.95$ (s, 2H, H4, and H4' $)$, 7.46-8.20 (m, 20H, Ar-H); UV-Vis $\left(\lambda_{\max }, \mathrm{nm}\right)$ : 385; Anal. Calculated for $\mathrm{C}_{42} \mathrm{H}_{22} \mathrm{Br}_{2} \mathrm{Cl}_{4} \mathrm{~N}_{8} \mathrm{O}_{8} \mathrm{Te}: \mathrm{C} 42.18$, H 1.85, N 9.37 Found: C 42.20, H 1.87, N 9.37\%.

Bis[(2-(3-(4-methylphenyl)-5-(2-chlorophenyl)-1H-pyrazol-1-yl)-3,5-dinitrophenyl)]tellurium dichloride (23)

Yellow crystalline solid; Yield: 73\%; M.p.: $104-106^{\circ} \mathrm{C}$; Molar conductance $\left(\Lambda_{\mathrm{m}}, \mathrm{ohm}^{-1} \mathrm{~cm}^{-1} \mathrm{~mol}^{-1}\right)$ : 21; $\mathrm{R}_{\mathrm{f}}$ (ethyl acetate-n-hexane) $=0.58$; FTIR $(\mathrm{KBr}) \mathrm{cm}^{-1}: 3063 \mathrm{w}, 2927,1600 \mathrm{~s}, 1570 \mathrm{~s}, 1485 \mathrm{~s}, 1438 \mathrm{~m}$, $1323 \mathrm{~s}, 1273 \mathrm{~m}, 1211 \mathrm{~m}, 1172 \mathrm{~m}, 1064 \mathrm{~s}, 1007 \mathrm{~m}, 976 \mathrm{~s}, 820 \mathrm{~m}, 756 \mathrm{~m}, 709 \mathrm{~m}, 687 \mathrm{~m}, 582 \mathrm{~m}, 536 \mathrm{~m}$; ${ }^{1} \mathrm{H}$ NMR (500 MHz, DMSO- $\left.d_{6}, \delta / \mathrm{ppm}\right): 2.06\left(\mathrm{~s}, 6 \mathrm{H}, 2 \mathrm{CH}_{3}\right) ; 5.92\left(\mathrm{~s}, 2 \mathrm{H}, \mathrm{H} 4\right.$, and $\left.\mathrm{H}^{\prime}\right), 7.45-8.20(\mathrm{~m}, 20 \mathrm{H}$, Ar-H); UV-Vis $\left(\lambda_{\max }, \mathrm{nm}\right)$ : 385; Anal. Calculated for $\mathrm{C}_{44} \mathrm{H}_{28} \mathrm{Cl}_{4} \mathrm{~N}_{8} \mathrm{O}_{8} \mathrm{Te}$ : C 49.57, H 2.65, N 10.51, Found: C 49.61, H 2.69, N 10.58\%.

Bis[(2-(3-(4-methoxyphenyl)-5-(2-chlorophenyl)-1H-pyrazol-1-yl)-3,5-dinitrophenyl)]tellurium dichloride (24)

Light-yellowish crystalline solid; Yield: $78 \%$; M.p.: $181-183{ }^{\circ} \mathrm{C}$; Molar conductance $\left(\Lambda_{\mathrm{m}}, \mathrm{ohm}^{-1} \mathrm{~cm}^{-1} \mathrm{~mol}^{-1}\right)$ : 26; Rf (ethyl acetate-n-hexane) $=0.60$; FTIR $(\mathrm{KBr}) \mathrm{cm}^{-1}: 3063 \mathrm{w}, 2928 \mathrm{w}$, $2843 \mathrm{w}, 1604 \mathrm{~s}, 1570 \mathrm{~s}, 1521 \mathrm{~m}, 1465 \mathrm{~m}, 1431 \mathrm{~m}, 1330 \mathrm{~s}, 1261 \mathrm{~m}, 1226 \mathrm{~m}, 1180 \mathrm{~m}, 1033 \mathrm{~m}, 1014 \mathrm{~m}, 976 \mathrm{~s}$, $829 \mathrm{~m}, 758 \mathrm{~m}, 682 \mathrm{~m}, 578 \mathrm{~m} ;{ }^{1} \mathrm{H}$ NMR $\left(500 \mathrm{MHz}\right.$, DMSO- $\left.d_{6}, \delta / \mathrm{ppm}\right): 3.86\left(\mathrm{~s}, 6 \mathrm{H}, 2 \mathrm{OCH}_{3}\right), 5.80(\mathrm{~s}, 2 \mathrm{H}, 2 \mathrm{H} 4)$, 7.45-8.20 (m, 20H, Ar-H); UV-Vis $\left(\lambda_{\max }, \mathrm{nm}\right)$ : 385; Anal. Calculated for $\mathrm{C}_{44} \mathrm{H}_{28} \mathrm{Cl}_{4} \mathrm{~N}_{8} \mathrm{O}_{10}$ Te: $\mathrm{C} 48.12, \mathrm{H} 2.57$, N 10.20, Found: C 48.18, H 2.62, N 10.20\%.

\subsubsection{General Method for the Preparation of Diaryl Tellurium Diiodides}

Bis[(2-(3-(4-substitutedphenyl)-5-(2-chlorophenyl)-1H-pyrazol-1-yl)-3,5-dinitrophenyl)]tellurium diiodides 25-27

A solution of iodine $(0.10 \mathrm{~g}, 0.78 \mathrm{mmol})$ in $10 \mathrm{~mL}$ of ethanol added to a solution of diaryl tellurides compounds 13, 14, or $15(0.78 \mathrm{mmol})$ in $20 \mathrm{~mL}$ ethanol with stirring at room temperature for $30 \mathrm{~min}$ gave a brown solid of compounds 25,26 , and 27 , respectively.

Bis[(2-(3-(4-bromophenyl)-5-(2-chlorophenyl)-1H-pyrazol-1-yl)-3,5-dinitrophenyl)]tellurium diiodides (25)

Yellowish brown crystalline solid; Yield: $73 \%$; M.p.: $113-115{ }^{\circ} \mathrm{C}$; Molar conductance $\left(\Lambda_{\mathrm{m}}, \mathrm{ohm}^{-1} \mathrm{~cm}^{-1} \mathrm{~mol}^{-1}\right): 27 ; \mathrm{R}_{\mathrm{f}}$ (ethyl acetate-n-hexane) $=0.66$; FTIR $(\mathrm{KBr}) \mathrm{cm}^{-1}: 3059 \mathrm{w}, 1604 \mathrm{~s}$, $1523 \mathrm{~m}, 1469 \mathrm{~m}, 1438 \mathrm{~m}, 1334 \mathrm{~s}, 1311 \mathrm{~m}, 1269 \mathrm{~m}, 1211 \mathrm{~m}, 1010 \mathrm{~m}, 972 \mathrm{~m}, 860 \mathrm{~m}, 748$ m, 721 m, $686 \mathrm{~m}$, 655; ${ }^{1} \mathrm{H}$ NMR (500 MHz, DMSO- $\left.d_{6}, \delta / \mathrm{ppm}\right): 5.95$ (s, 2H, H4 and H4'), 7.46-8.20 (m, 20H, Ar-H); UV-Vis $\left(\lambda_{\max }, \mathrm{nm}\right): 390$; Anal. Calculated for $\mathrm{C}_{42} \mathrm{H}_{22} \mathrm{I}_{2} \mathrm{Br}_{2} \mathrm{Cl}_{2} \mathrm{~N}_{8} \mathrm{O}_{8}$ Te: C 27.52, H 1.68, N 5.84 Found: C 27.60, H 1.71, N 5.91\%.

Bis[(2-(3-(4-methylphenyl)-5-(2-chlorophenyl)-1H-pyrazol-1-yl)-3,5-dinitrophenyl)]tellurium diiodides (26)

Yellowish brown crystalline solid; Yield: 77\%; M.p.: $80-82{ }^{\circ} \mathrm{C}$; Molar conductance $\left(\Lambda_{\mathrm{m}}, \mathrm{ohm}^{-1} \mathrm{~cm}^{-1} \mathrm{~mol}^{-1}\right): 21 ; \mathrm{R}_{\mathrm{f}}($ ethyl acetate-n-hexane $)=0.66 ;$ FTIR $(\mathrm{KBr}) \mathrm{cm}^{-1}: 3059 \mathrm{~m}, 2920 \mathrm{w}$, $1604 \mathrm{~s}, 1523 \mathrm{~m}, 1462 \mathrm{~m}, 1438 \mathrm{~m}, 1334,1311 \mathrm{~s}, 1269 \mathrm{~m}, 1211 \mathrm{~s}, 1037 \mathrm{~m}, 1010 \mathrm{~m}, 972 \mathrm{~m}, 860,748 \mathrm{~s}, 721 \mathrm{~m}$, $585 \mathrm{~m}, 655 \mathrm{~m}, 578 \mathrm{~m}, 528 \mathrm{~m}, 447 \mathrm{~m} ;{ }^{1} \mathrm{H}$ NMR (500 MHz, DMSO- $\left.d_{6}, \delta / \mathrm{ppm}\right): 2.06\left(\mathrm{~s}, 6 \mathrm{H}, 2 \mathrm{CH}_{3}\right)$; $5.92\left(\mathrm{~s}, 2 \mathrm{H}, \mathrm{H} 4\right.$, and $\left.\mathrm{H} 4^{\prime}\right), 7.45-8.20(\mathrm{~m}, 20 \mathrm{H}, \mathrm{Ar}-\mathrm{H})$; UV-Vis $\left(\lambda_{\max }, \mathrm{nm}\right): 395$; Anal. Calculated for $\mathrm{C}_{44} \mathrm{H}_{28} \mathrm{I}_{2} \mathrm{Cl}_{2} \mathrm{~N}_{8} \mathrm{O}_{8}$ Te: C 36.59, H 1.61, N 8.13, Found: C 37.04, H 1.66, N 8.15 \%.

Bis[(2-(3-(4-methoxyphenyl)-5-(2-chlorophenyl)-1H-pyrazol-1-yl)-3,5-dinitrophenyl)]tellurium diiodides (27)

Yellowish brown crystalline solid; Yield: 66\%; M.p.: 93-95 ${ }^{\circ} \mathrm{C}$; Molar conductance $\left(\Lambda_{\mathrm{m}}, \mathrm{ohm}^{-1} \mathrm{~cm}^{-1} \mathrm{~mol}^{-1}\right): 19$; Rf (ethyl acetate-n-hexane) $=0.60 ;$ FTIR $(\mathrm{KBr}) \mathrm{cm}^{-1}: 3056 \mathrm{w}, 2928 \mathrm{w}$, $2840 \mathrm{w}, 1600 \mathrm{~s}, 1572 \mathrm{~s}, 1521 \mathrm{~m}, 1465 \mathrm{~m}, 1430 \mathrm{~m}, 1334 \mathrm{~s}, 1260 \mathrm{~m}, 1226 \mathrm{~m}, 1180 \mathrm{~m}, 1032 \mathrm{~m}, 1016 \mathrm{~m}, 976 \mathrm{~s}$, 
829 m, 758 m, 682 m, 577 m; ${ }^{1} \mathrm{H}$ NMR (500 MHz, DMSO-d 6 , $\left./ \mathrm{ppm}\right): 3.83$ (s, 6H, 2OCH $), 5.82$ (s, 2H, H4, and $\left.\mathrm{H}^{\prime}\right)$, 7.46-8.22 (m, 20H, Ar-H); UV-Vis $\left(\lambda_{\text {max }}, \mathrm{nm}\right)$ : 390; Anal. Calculated for $\mathrm{C}_{44} \mathrm{H}_{28} \mathrm{I}_{4} \mathrm{Cl}_{2} \mathrm{~N}_{8} \mathrm{O}_{10} \mathrm{Te}$ C 42.31, H 2.26, N 8.97, Found: C 42.39, H 2.31, N 9.02\%.

\subsection{Determination of Antimicrobial Activity}

Compounds 1-27 were assayed against four different microorganisms: Gram-positive bacteria Staphylococcus aureus ATCC25923 and Candida albicans ATCC2091, and Gram-negative bacteria Escherichia coli ATCC25922 and Pseudomonas aeruginosa ATCC9027 by using the disk diffusion technique. Amoxicillin $(10 \mu \mathrm{g} / \mathrm{disc})$ was used as the standard drug. The agar well diffusion method was applied for the determination of the inhibition zone and minimum inhibitory concentration (MIC). The procedure used in this study was according to a previous method [4].

\section{Conclusions}

This work shows an efficient method for the synthesis of a series of new tellurated compounds derived from 1, 3, 5-trisubstituted-pyrazole derivatives for the first time. The compounds that possess pharmacophores such as bromo-, methoxy- and methyl-substituents with lipophilic properties appeared to have the greatest antimicrobial activity. The diaryl tellurides 13, 14, and 15 were highly active against the organisms employed among all synthesized compounds. Compounds 13-15 possess more potent activity against the bacteria than control (amoxicillin), which makes them promising drugs in the future.

Supplementary Materials: The following are available online, Figure S1: ${ }^{1} \mathrm{H}$ NMR spectrum of 2-(3-(4-Bromophenyl)5-(2-chlorophenyl)-1H-pyrazol-1-yl)-3,5-dinitrophenyl) mercury (II) chloride (1), Figure S2: ${ }^{13} \mathrm{C}$ NMR spectrum of 2-(3-(4-Bromophenyl)-5-(2-chlorophenyl)-1H-pyrazol-1-yl)-3,5-dinitrophenyl) mercury (II) chloride (1), Figure S3: ${ }^{1} \mathrm{H}$ NMR spectrum of 2-(5-(2-Chlorophenyl)-3-(4-methyl phenyl)-1H-pyrazol-1-yl)-3,5-dinitro phenyl) mercury(II) chloride (2), Figure S4: ${ }^{13} \mathrm{C}$ NMR spectrum of 2-(5-(2-Chlorophenyl)-3-(4-methyl phenyl)-1H-pyrazol-1-yl)-3,5dinitro phenyl) mercury(II) chloride (2), Figure S5: ${ }^{1} \mathrm{H}$ NMR spectrum of 2-(5-(2-Chlorophenyl)-3-(4-methoxy phenyl)-1H-pyrazol-1-yl)-3,5-dinitrophenyl)mercury(II) chloride(3), Figure S6: ${ }^{13} \mathrm{C}$ NMR spectrum of 2-(5(2-Chlorophenyl)-3-(4-methoxyphenyl) -1H-pyrazol-1-yl)-3,5-dinitrophenyl)mercury(II) chloride (3), Figure S7: ${ }^{1} \mathrm{H}$ NMR spectrum of (2-(3-(4-Bromophenyl)-5-(2-chlorophenyl)-1H-pyrazol-1-yl)-3,5-dinitrophenyl)tellurium tribromide (4), Figure S8: ${ }^{13} \mathrm{C}$ NMR spectrum of (2-(3-(4-Bromophenyl)-5-(2-chlorophenyl)-1H -pyrazol-1-yl) -3,5-dinitrophenyl)tellurium tribromide (4)., Figure S9: ${ }^{1} \mathrm{H}$ NMR spectrum of (2(5-(2-Chlorophenyl)-3-(4-methylphenyl)-1H-pyrazol-1-yl)-3,5-dinitrophenyl)tellurium tribromide (5), Figure S10: ${ }^{13} \mathrm{C}$ NMR spectrum of (2-(5-(2-Chlorophenyl)-3-(4-methylphenyl)-1H-pyrazol-1-yl)-3,5-dinitrophenyl) tellurium tribromide (5), Figure S11: ${ }^{1} \mathrm{H}$ NMR spectrum of (2-(5-(2-Chlorophenyl)- 3-(4-methoxyphenyl)1H-pyrazol-1-yl) -3,5dinitrophenyl) tellurium tribromide (6), Figure S12: C NMR spectrum of (2-(5-(2-Chlorophenyl)-3-(4-methoxyphenyl)-1H-pyrazol-1-yl)-3,5dinitrophenyl)tellurium tribromide (6), Figure S13: ${ }^{1} \mathrm{H}$ NMR spectrum of Bis[(2-(3-(4-bromophenyl)-5-(2-chlorophenyl)-1H-pyrazol-1-yl)-3,5-dinitrophenyl)] tellurium dibromide (7), Figure S14: ${ }^{13} \mathrm{C}$ NMR spectrum of Bis[(2-(3-(4-bromophenyl)-5-(2-chlorophenyl)-1Hpyrazol-1-yl)-3,5-dinitrophenyl)]tellurium dibromide (7), Figure S15: ${ }^{1} \mathrm{H}$ NMR spectrum of Bis[(2-(5-(2 -Chlorophenyl)-3-(4-methylphenyl)-1H-pyrazol-1-yl)-3,5-dinitrophenyl)] tellurium dibromide (8), Figure S16: ${ }^{13} \mathrm{C}$ NMR spectrum of Bis[(2-(5-(2-Chlorophenyl)-3-(4-methylphenyl)-1H-pyrazol-1-yl)-3,5-dinitrophenyl)]tellurium dibromide (8), Figure S17: ${ }^{1} \mathrm{H}$ NMR spectrum of Bis[2-(5-(2-Chlorophenyl)-3-(4-methoxyphenyl)-1H-pyrazol-1-yl)3,5dinitro phenyl)] tellurium dibromide (9), Figure S18: ${ }^{13} \mathrm{C}$ NMR spectrum of Bis[2-(5-(2-Chlorophenyl)-3(4-methoxyphenyl)-1H-pyrazol-1-yl)-3,5dinitro phenyl)]tellurium dibromide (9), Figure S19: ${ }^{1} \mathrm{H}$ NMR spectrum of Bis[(2-(3-(4-bromophenyl)-5-(2-chlorophenyl)-1H-pyrazol-1-yl)-3,5-dinitro phenyl)] ditelluride (10), Figure S20: ${ }^{13} \mathrm{C}$ NMR spectrum of Bis[(2-(3-(4-bromophenyl)-5-(2-chlorophenyl)-1H-pyrazol-1-yl)-3,5-dinitrophenyl)] ditelluride (10), Figure S21: ${ }^{1}$ H NMR spectrum of Bis[(2-(5-(2-Chlorophenyl)-3-(4-methylphenyl)-1H-pyrazol-1-yl)-3,5-dinitrophenyl)] ditelluride (11), Figure S22: ${ }^{13}$ C NMR spectrum of bis[(2-(5-(2-Chlorophenyl)-3-(4-methylphenyl)-1H-pyrazol-1-yl)-3,5dinitrophenyl)] ditelluride (11), Figure S23: ${ }^{1} \mathrm{H}$ NMR spectrum of Bis[(2-(5-(2-Chlorophenyl) -3-(4-methoxyphenyl)-1H-pyrazol-1-yl)-3,5dinitro phenyl)] ditelluride (12), Figure S24: ${ }^{13} \mathrm{C}$ NMR spectrum of Bis[(2-(5-(2-Chlorophenyl)-3-(4-methoxyphenyl)-1H-pyrazol-1-yl)-3,5dinitro phenyl)] ditelluride (12), Figure S25: ${ }^{1} \mathrm{H}$ NMR spectrum of Bis[(2-(3-(4-bromophenyl)-5-(2-chlorophenyl)-1H-pyrazol-1-yl)-3,5-dinitrophenyl)] telluride (13), Figure S26: C NMR spectrum of bis[(2-(3-(4-bromophenyl)-5-(2-chlorophenyl)-1H-pyrazol-1-yl)-3,5-dinitrophenyl)] telluride (13), Figure S27: ${ }^{1} \mathrm{H}$ NMR spectrum of Bis[(2-(5-(2-Chlorophenyl)-3-(4-methylphenyl)-1H-pyrazol-1-yl)-3,5dinitrophenyl)] telluride (14), Figure S28: ${ }^{13} \mathrm{C}$ NMR spectrum of Bis[(2-(5-(2-Chlorophenyl) 
-3-(4-methylphenyl)-1H-pyrazol-1-yl)-3,5-dinitrophenyl)] telluride (14), Figure S29: ${ }^{1} \mathrm{H}$ NMR spectrum of bis[(2-(5-(2-Chlorophenyl)-3-(4-methoxyphenyl)-1H-pyrazol-1-yl)-3,5-dinitrophenyl)] telluride (15), Figure S30: ${ }^{13} \mathrm{C}$ NMR spectrum of Bis[(2-(5-(2-Chlorophenyl)-3-(4-methoxyphenyl)-1H-pyrazol-1-yl)-3,5-dinitrophenyl)] telluride (15), Figure S31: ${ }^{1} \mathrm{H}$ NMR spectrum of (2-(3-(4-Bromophenyl)-5-(2-chlorophenyl)-1H-pyrazol-1-yl)-3,5dinitrophenyl)tellurium trichloride (16), Figure S32: ${ }^{13} \mathrm{C}$ NMR spectrum of (2-(3-(4-Bromophenyl)-5(2-chlorophenyl)-1H-pyrazol-1-yl)-3,5-dinitrophenyl)tellurium trichloride (16), Figure S33: ${ }^{1} \mathrm{H} \quad \mathrm{NMR}$ spectrum of (2-(3-(4-Methylphenyl)-5-(2-chlorophenyl)-1H-pyrazol-1-yl)-3,5-dinitrophenyl)tellurium trichloride (17), Figure S34: ${ }^{13} \mathrm{C}$ NMR spectrum of (2-(3-(4-Methylphenyl)-5-(2-chlorophenyl)-1H-pyrazol-1-yl)-3,5dinitrophenyl) tellurium trichloride (17), Figure S35: ${ }^{1} \mathrm{H}$ NMR spectrum of (2-(3-(4-Methoxyphenyl)5-(2-chlorophenyl)-1H-pyrazol-1-yl)-3,5-dinitrophenyl)tellurium trichloride (18), Figure S36: ${ }^{13} \mathrm{C}$ NMR spectrum of (2-(3-(4-Methoxyphenyl)-5-(2-chlorophenyl)-1H-pyrazol-1-yl)-3,5-dinitrophenyl) tellurium trichloride (18), Figure S37: ${ }^{1} \mathrm{H}$ NMR spectrum of (2-(3-(4-Bromophenyl)-5-(2-chlorophenyl)-1H-pyrazol-1-yl)-3,5-dinitrophenyl)tellurium triiodide (19), Figure S38: C NMR spectrum of (2-(3-(4-Bromophenyl)-5-(2-chlorophenyl)-1H-pyrazol-1-yl)-3,5 -dinitrophenyl)tellurium triiodide (19), Figure S39: ${ }^{1} \mathrm{H}$ NMR spectrum of (2-(3-(4-Methylphenyl)-5-(2-chlorophenyl)1H-pyrazol-1-yl)-3,5-dinitrophenyl)tellurium triiodide $(20)$, Figure $\mathrm{S} 40$ : ${ }^{13} \mathrm{C}$ NMR spectrum of (2-(3-(4-Methylphenyl)-5-(2-chlorophenyl)-1H-pyrazol-1-yl)-3,5-dinitrophenyl)tellurium triiodide (20), Figure S41: ${ }^{1} \mathrm{H}$ NMR spectrum of (2-(3-(4-Methoxyphenyl)-5-(2-chlorophenyl)-1H-pyrazol-1-yl)-3,5-dinitrophenyl)tellurium triiodide (21), Figure S42: ${ }^{13} \mathrm{C}$ NMR spectrum of (2-(3-(4-Methoxyphenyl)-5-(2-chlorophenyl)-1H-pyrazol-1-yl)-3,5 -dinitrophenyl)tellurium triiodide (21), Figure S43: ${ }^{1} \mathrm{H}$ NMR spectrum of Bis[(2-(3-(4-bromophenyl) -5-(2-chlorophenyl)-1H-pyrazol-1-yl)-3,5-dinitro phenyl)]tellurium dichloride (22), Figure S44: ${ }^{13} \mathrm{C}$ NMR spectrum of bis[(2-(3-(4-bromophenyl)-5-(2-chlorophenyl)-1H-pyrazol-1-yl)-3,5-dinitrophenyl)]tellurium dichloride (22), Figure S45: ${ }^{1}$ H NMR spectrum of Bis[(2-(3-(4-methylphenyl)-5-(2-chlorophenyl)-1H-pyrazol-1-yl)-3,5-dinitro phenyl)]tellurium dichloride (23), Figure $\mathrm{S} 46:{ }^{13} \mathrm{C}$ NMR spectrum of Bis[(2-(3-(4-methylphenyl) -5-(2-chlorophenyl)-1H-pyrazol-1-yl)-3,5-dinitrophenyl)]tellurium dichloride (23), Figure S47: ${ }^{1} \mathrm{H}$ NMR spectrum of Bis[(2-(3-(4-methoxyphenyl)-5-(2-chlorophenyl)-1H-pyrazol-1-yl)-3,5-dinitro phenyl)]tellurium dichloride (24), Figure S48: ${ }^{13} \mathrm{C}$ NMR spectrum of Bis[(2-(3-(4-methoxyphenyl)5-(2-chlorophenyl)-1H-pyrazol-1-yl)-3,5-dinitrophenyl)]tellurium dichloride (24), Figure S49: ${ }^{1} \mathrm{H} \quad \mathrm{NMR} \quad$ spectrum of Bis[(2-(3-(4-bromophenyl)-5-(2-chlorophenyl)-1H-pyrazol-1-yl)-3,5-dinitro phenyl)]tellurium diiodides (25), Figure S50: ${ }^{13} \mathrm{C}$ NMR spectrum of Bis[(2-(3-(4-bromophenyl)5-(2-chlorophenyl)-1H-pyrazol-1-yl)-3,5-dinitrophenyl)]tellurium diiodides (25), Figure S51: ${ }^{1} \mathrm{H}$ NMR spectrum of Bis[(2-(3-(4-methylphenyl)-5-(2-chlorophenyl)-1H-pyrazol-1-yl)-3,5-dinitro phenyl)] tellurium diiodides (26), Figure $\quad 552: \quad{ }^{13} \mathrm{C}$ NMR spectrum of Bis[(2-(3-(4-methylphenyl)-5-(2-chlorophenyl)-1H-pyrazol-1-yl)-3,5-dinitrophenyl)]tellurium diiodides (26), Figure S53: ${ }^{1} \mathrm{H}$ NMR spectrum of Bis[(2-(3-(4-methoxyphenyl)-5- (2-chlorophenyl)-1H-pyrazol-1-yl)-3,5-dinitro phenyl)] tellurium diiodides (27), Figure S54: ${ }^{13} \mathrm{C}$ NMR spectrum of bis[(2-(3-(4-methoxyphenyl)5-(2-chlorophenyl)-1H-pyrazol-1-yl)-3,5-dinitrophenyl)]tellurium diiodides (27), Figure S55: IR spectrum of 2-(3-(4-bromophenyl)-5-(2-chlorophenyl)-1H-pyrazol-1-yl)-3,5-dinitrophenyl) mercury (II) chloride (1), Figure S56: IR spectrum of 2-(5-(2-chlorophenyl)-3-(4-methoxyphenyl)-1H-pyrazol-1-yl)-3,5dinitrophenyl)mercury(II) chloride (3), Figure S57: IR spectrum of (2-(3-(4-bromophenyl)5-(2-chlorophenyl)-1H-pyrazol-1-yl)-3,5-dinitrophenyl)tellurium tribromide (4), Figure S58: IR spectrum of (2-(5-(2-chlorophenyl)-3-(4-methoxyphenyl)-1H-pyrazol-1-yl)-3,5dinitrophenyl)tellurium tribromide (6), Figure S59: IR spectrum of bis[(2-(3-(4-bromophenyl)-5-(2-chlorophenyl)-1H-pyrazol-1-yl)-3,5-dinitrophenyl)]tellurium dibromide (7), Figure S60: IR spectrum of bis[(2-(5-(2-chlorophenyl)-3-(4-methylphenyl)-1H-pyrazol-1-yl)-3,5-dinitro phenyl)] tellurium dibromide (8), Figure S61: IR spectrum of bis[(2-(5-(2-chlorophenyl)-3-(4-methylphenyl)- $1 \mathrm{H}-$ pyrazol-1-yl)-3,5-dinitrophenyl)] ditelluride (11), Figure S62: IR spectrum of bis[(2-(5-(2-chlorophenyl)-3 -(4-methoxyphenyl)-1H-pyrazol-1-yl)-3,5dinitro phenyl)] ditelluride (12), Figure S63: IR spectrum of bis[(2-(3-(4-bromophenyl)-5-(2-chlorophenyl)-1H-pyrazol-1-yl)-3,5-dinitro phenyl)] telluride (13), Figure S64: IR spectrum of bis[(2-(5-(2-chlorophenyl)-3-(4-methoxyphenyl)-1H-pyrazol-1-yl)-3,5- dinitrophenyl)] telluride (15), Figure S65: IR spectrum of (2-(3-(4-methylphenyl)-5-(2-chlorophenyl)-1H-pyrazol-1-yl)-3,5-dinitrophenyl)tellurium trichloride (17), Figure S66: IR spectrum of bis[(2-(3-(4-bromophenyl)-5-(2-chlorophenyl)-1H-pyrazol-1-yl)-3,5dinitrophenyl)]tellurium dichloride (22), Figure S67: IR spectrum of bis[(2-(3-(4-methylphenyl)5-(2-chlorophenyl)-1H-pyrazol-1-yl)-3,5-dinitrophenyl)]tellurium dichloride (23), Figure S68: IR spectrum of (2-(3-(4-methylphenyl)-5-(2-chlorophenyl)-1H-pyrazol-1-yl)-3,5-dinitro phenyl) tellurium triiodide (20), Figure S69: IR spectrum of bis[(2-(3-(4-methoxyphenyl)-5-(2-chlorophenyl)-1H- pyrazol-1-yl)-3,5-dinitrophenyl)]tellurium diiodides (27). 
Author Contributions: Conceptualization, A.A.A.-F. and M.Y.Y.; methodology, A.B.S.; software, A.A.A.-F.; validation, A.A.A.-F., M.Y.Y. and A.B.S.; formal analysis, A.A.A.-F.; investigation, A.A.A.-F.; resources, A.A.A.-F.; data curation, A.A.A.-F.; writing-original draft preparation, A.B.S.; writing-review and editing, A.A.A.-F.; visualization M.Y.Y.; supervision, A.A.A.-F.; project administration, A.A.A.-F.; funding acquisition A.A.A.-F. All authors (A.A.A.-F., A.B.S. and M.Y.Y.) have read and agreed to the published version of the manuscript.

Funding: This research received no external funding.

Acknowledgments: Authors are thankful to the Department of Chemistry, College of Science, University of Basrah for providing the laboratory facilities.

Conflicts of Interest: The authors declare no conflict of interest.

\section{References}

1. Fustero, S.; Simon-Fuentes, A.; Sanz-Cervera, J.F. Recent advances in the synthesis of pyrazoles: A review. Org. Prep. Proced. Int. 2009, 41, 253-290. [CrossRef]

2. Karrouchi, K.; Radi, S.; Ramli, Y.; Taoufik, J.; Mabkhot, Y.N.; Al-aizari, M.; Ansar, M. Synthesis and pharmacological activities of pyrazole derivatives: A review. Molecules 2018, 23, 134. [CrossRef] [PubMed]

3. Fustero, S.; Sánchez-Roselló, M.; Barrio, P.; Simón-Fuentes, A. From 2000 to mid-2010: A fruitful decade for the synthesis of pyrazoles. Chem. Rev. 2011, 111, 6984-7034. [CrossRef] [PubMed]

4. Sharshira, M.E.; Hamada, N.M. Synthesis and pharmacological activities of pyrazole derivatives: A review. Molecules 2012, 17, 4962-4971. [CrossRef] [PubMed]

5. Abdelhamid, A.O.; Zohidi, H.F.; Sallam, M.M.M.; Ahmed, N.A. Synthesis of some new pyrrolidino[3,4-c]pyrazolines, pyrazoles, and pyrazolo[3,4- d] pyridazines. Molecules 2000, 5, 967-973. [CrossRef]

6. Bhasin, K.K.; Pundir, S.; Neogy, S.; Mehta, D.; Mehta, S.K. Synthesis, characterization and structural aspect of some symmetrical organotellurium halides based on Bis(2-(3,5-dimethyl-1H-pyrazole-1-yl)ethyl)telluride. Phosphorus Sulfur Silicon Relat. Elem. 2018, 193, 273-279. [CrossRef]

7. Chandrasekhar, V.; Kumar, A.; Pandey, M.D.; Metre, R.K. Telluroxane-supported coordination ligands: Synthetic and structural aspects. Polyhedron 2013, 52, 1362-1368. [CrossRef]

8. Nogueira, C.W.; Zeni, G.; Rocha, J.B.T. Organoselenium and organotellurium compounds: Toxicology and pharmacology. Chem. Rev. 2004, 104, 6255-6285. [CrossRef] [PubMed]

9. Reis de Sá, L.F.; Toledo, F.T.; Gonçalves, A.C.; Sousa, B.A.; dos Santos, A.A.; Brasil, P.F.; Duarte da Silva, V.A.; Tessis, A.C.; Ramos, J.A.; Carvalho, M.A.; et al. Synthetic organotellurium compounds sensitize drug-resistant Candida albicans clinical isolates to fluconazole. Antimicrob. Agents Chemother. 2017, 61, e01231-16. [CrossRef] [PubMed]

10. Reis de Sá, L.F.; Toledo, F.T.; de Sousa, B.A.; Goncalves, A.C.; Tessis, A.C.; Wendler, E.P.; Comasseto, J.V.; Dos Santos, A.A.; Ferreira-Pereira, A. Synthetic organotelluride compounds induce the reversal of Pdr5p-mediated fluconazole resistance in Saccharomyces cerevisiae. BMC Microbiol. 2014, 14, 201. [CrossRef] [PubMed]

11. Al-Rubaie, A.Z.; Al-Fregi, A.A.; Al-Jadaan, S.A.S. Synthesis of a new series of 2-(2-hydroxynaphthylazo)aryltellurium compounds. Phosphorus Sulfur Silicon Relat. Elem. 2011, 186, 115-124. [CrossRef]

12. Al-Fregi, A.A.; Al-Salami, B.K.; Al-Khazragie, Z.K.; Al-Rubaie, A.Z. Synthesis, characterization and antibacterial studies of some new tellurated azo compounds. Phosphorus Sulfur Silicon Relat. Elem. 2019, 194, 33-38. [CrossRef]

13. Sabti, A.B. Synthesis, Characterization and Microbial Activity Study of Some New Organotellurium Compounds. Ph.D. Thesis, University of Basrah, Basrah, Iraq, 2020.

14. Jimeno, M.L.; Jagerovic, N.; Elguero, J.; Junk, T.; Catallo, W.J. ${ }^{1} \mathrm{H}$ and ${ }^{13} \mathrm{C}$ NMR study of perdeuterated pyrazoles. Spectroscopy 1997, 13, 291-294. [CrossRef]

15. Tala, S.D.; Vekariya, P.B.; Ghetiya, R.M.; Dodiya, B.L.; Joshi, H.S. Synthesis and biological study of some new chalcone and pyrazole derivatives. Indian J. Chem. 2013, 52B, 807-809.

16. Saadon, H.L.; Ali, B.; Al-Fregi, A.A. Nonlinear optical properties of new organotellurium compounds containing azomethine and azo groups under CW laser illumination. Opt. Laser Technol. 2014, 58, 33-38. [CrossRef] 
17. Hockstedler, A.N.; Edjah, B.A.; Saajid, Z.; Azhar, S.Z.; Mendoza, H.; Brown, N.A.; Arrowood, H.B.; Clay, A.C.; Shah, A.B.; Duffek, G.M.; et al. ${ }^{13} \mathrm{C}$ NMR spectroscopy of heterocycles: 1-phenyl-3-aryl/t-butyl-5-arylpyrazoles. Heterocycl. Commun. 2017, 23, 125-131. [CrossRef]

18. Durust, Y.; Yildirum, M. Synthesis, UV-Vis spectra, and Hammett correlation of some novel bis(dihydropyrrolo[3,4-c]pyrazoles). Mon. Chem. 2010, 141, 961-973. [CrossRef]

19. Al-Fregi, A.A.; Mayada Abdulaa Adnan, M.A. Synthesis, characterization and analytical study of tellurated Schiff base of bis[2-(3-nitrobenzylideneamino)-5-nitrophenyl]telluride and its complexation reactions with Mn(II), Co(II) and Ni(II) ions. Eur. J. Chem. 2016, 7, 195-200. [CrossRef]

20. Al-Fregi, A.A.; Jaid, H.A.; Abdulsahib, H.T. Synthesis and characterization of some new organotellurium compounds based on quinolone. Eur. J. Chem. 2017, 8, 218-223. [CrossRef]

Sample Availability: Samples of the compounds are not available from the authors.

(C) 2020 by the authors. Licensee MDPI, Basel, Switzerland. This article is an open access article distributed under the terms and conditions of the Creative Commons Attribution (CC BY) license (http://creativecommons.org/licenses/by/4.0/). 